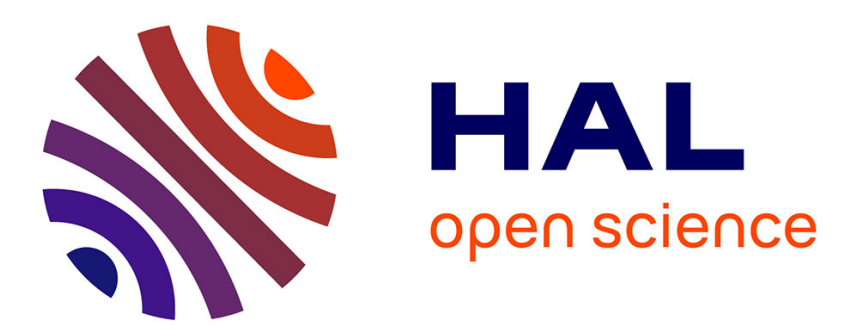

\title{
Analysis of degenerate mechanisms triggering finite-amplitude thermo-acoustic oscillations in annular combustors
}

\author{
Sandeep Murthy, Taraneh Sayadi, Vincent Le Chenadec, Peter J. Schmid, \\ Daniel J Bodony
}

\section{To cite this version:}

Sandeep Murthy, Taraneh Sayadi, Vincent Le Chenadec, Peter J. Schmid, Daniel J Bodony. Analysis of degenerate mechanisms triggering finite-amplitude thermo-acoustic oscillations in annular combustors. Journal of Fluid Mechanics, 2019, 881, pp.384-419. 10.1017/jfm.2019.720 • hal-03011379

\author{
HAL Id: hal-03011379 \\ https://hal.science/hal-03011379
}

Submitted on 23 Nov 2020

HAL is a multi-disciplinary open access archive for the deposit and dissemination of scientific research documents, whether they are published or not. The documents may come from teaching and research institutions in France or abroad, or from public or private research centers.
L'archive ouverte pluridisciplinaire HAL, est destinée au dépôt et à la diffusion de documents scientifiques de niveau recherche, publiés ou non, émanant des établissements d'enseignement et de recherche français ou étrangers, des laboratoires publics ou privés. 


\title{
Analysis of degenerate mechanisms triggering finite amplitude thermo-acoustic oscillations in annular combustors
}

\author{
S.R. Murthy ${ }^{1} \dagger$, T. Sayadi ${ }^{2}$, V. LeChenadec ${ }^{3}$, Peter J. Schmid ${ }^{4}$ and \\ D.J. Bodony ${ }^{1}$ \\ ${ }^{1}$ Department of Aerospace Engineering, University of Illinois at Urbana-Champaign, Urbana \\ 61820, USA. \\ ${ }^{2}$ Jean de Rond d'Alembert institute, Sorbonne University, Paris, France \\ ${ }^{3}$ Multi Scale Modeling and Simulation Laboratory, University of Paris-Est, Marne-la-Valle, \\ France \\ ${ }^{4}$ Department of Mathematics, Imperial College London, London SW7 2AZ, U.K
}

(Received $\mathrm{xx}$; revised $\mathrm{xx}$; accepted $\mathrm{xx}$ )

A simplified model is introduced to study finite-amplitude thermo-acoustic oscillations in $N$-periodic annular combustion devices. Such oscillations yield undesirable effects and can be triggered by a positive feedback between heat-release and pressure fluctuations. The proposed model, comprised of the governing equations linearized in the acoustic limit, and with each burner modeled as a one-dimensional system with acoustic damping and a compact heat source, is used to study the instability caused by cross-sector coupling. The coupling between the sectors is included by solving the one-dimensional acoustic jump conditions at the locations where the burners are coupled to the annular chambers of the combustion device. The analysis takes advantage of the block-circulant structure of the underlying stability equations to develop an efficient methodology to describe the onset of azimuthally-synchronized motion. A modal analysis reveals the dominance of large-scale instabilities, while a non-modal analysis reveals a strong response to harmonic excitation at forcing frequencies far from the eigenfrequencies, when the overall system is linearly stable. In all presented cases, large-scale, azimuthally synchronized motion is observed. The relevance of the non-modal response is further emphasized by demonstrating the subcritical nature of the system's Hopf point via an asymptotic expansion of a nonlinear model representing the compact heat source within each burner.

\section{Introduction}

Energy conversion devices with high power densities, such as gas turbines, can exhibit thermo-acoustic instabilities (Lieuwen \& Yang 2005; Culick \& Kuentzmann 2006), caused by a resonant feedback between acoustic radiation and combustion processes (Candel 1992; Dowling 1995; Sattelmayer \& Polifke 2003). The driving mechanism behind such instabilities was recognized by Lord Rayleigh (1878) who observed amplifying oscillations when pressure and heat-release fluctuations were in phase and caused positive feedback effects.

Thermo-acoustic instabilities severely affect the lifetime, performance and operational safety of energy conversion devices. In an effort to meet reduced mono-nitrogen oxide $\left(\mathrm{NO}_{\mathrm{x}}\right)$ and soot emissions mandated by environmental regulatory policies, highperformance engines often operate in the lean regime, where thermo-acoustic instabilities

$\dagger$ Email address for correspondence: srmurth2@illinois.edu 
distinctly prevail (Harrje 1972; Culick 1988). The additional absence of bypass air in convectively-cooled combustion systems yields a decrease of acoustic damping and an increase in pulsation amplitudes, further increasing the likelihood of instability. Hence, a combination of lean-burn parameters and convectively cooled combustion chambers has made energy conversion devices more prone to combustion-driven oscillations, or thermo-acoustic instabilities. These oscillations typically trigger the lowest-frequency eigenmodes (Sensiau et al. 2008). As the radial and longitudinal dimensions of most combustion chambers are shorter than their azimuthal dimension, these lowest-frequency modes manifest themselves as azimuthal modes (Salas 2013).

Experimental investigations on full-scale engines are difficult and expensive (Poinsot et al. 1987; Lee \& Anderson 1999; Lee \& Lieuwen 2003). Therefore, simplified configurations have been used to study both longitudinal and azimuthal instabilities. Earlier studies by Candel et al. (1988, 2004) and Ducruix et al. (2005) focused on longitudinal combustion instabilities, while, more recently, azimuthal modes were studied in a simplified, annular Rijke-tube configuration, using heating grids as the unsteady heat source (Moeck et al. 2010; Gelbert et al. 2012). Worth \& Dawson $(2013 a, b)$ examined an annular configuration with swirled, premixed flames to study the interaction between the flames and the mean swirl as well as changes to the stability and nature of azimuthal modes. However, swirling turbulent flames can compromise the study of the thermo-acoustic phenomena; therefore, Bourgouin et al. (2015) specifically designed an annular combustion chamber to avoid such complexities. Experiments of this type provide invaluable insight, but require significant cost and effort (Dowling 1995; Kopitz et al. 2005; Nicoud \& Poinsot 2005), motivating analytical and numerical approaches, ranging from theoretical models, via low-order network methods, to full-scale Large Eddy Simulations (LES). The cost and complexity of each approach increases with the level of fidelity and detailed insight it provides.

Three-dimensional numerical simulations, such as LES, are capable of reproducing combustion instabilities in complex geometries by accounting for the relevant mechanisms with minimal modeling assumptions. Recent studies by Staffelbach et al. (2009) or Wolf et al. (2012) have successfully simulated azimuthal instabilities in real gas-turbine combustion chambers using LES. Although computationally demanding, LES can predict and reproduce combustion instabilities, but can provide only limited insight into means to identify and control the unstable modes that arise (Dowling 1995; Kopitz et al. 2005; Nicoud \& Poinsot 2005). Hence, low-order and low-complexity models are needed to guide computationally intensive simulations or experiments.

The need to investigate combustion processes across multiple, varying parameters, to determine the manner in which they affect instabilities, and to explore potential control strategies, has generated renewed interest in analytical techniques (Clavin et al. 1990; Dowling 1995; Poinsot \& Veynante 2005; George \& Sujith 2011), where the nature and structure of azimuthal modes in annular combustion chambers have been clarified without computational simulations (Parmentier et al. 2012). Using simple geometric configurations, studies of this kind provide a mechanistic point-of-view and guide the interpretation of thermo-acoustic instabilities in more complex simulations. However, these methods rely on hypotheses that have to be satisfied in order to simplify the problem; hence only configurations and geometries of low complexity can be studied.

Network models can be used to explore moderately complex geometries and configurations, where analytical studies fail. Using this approach, the actual geometry is decomposed into a set of lumped acoustic elements and compact heat sources. Each element is modeled by means of a linear transfer function (Polifke et al. 2001; Stow \& Dowling 2001; Poinsot \& Veynante 2005) and composed into a more complex system. 
In a recent investigation by Parmentier et al. (2012), a semi-analytical approach with a one-dimensional network model was used to study azimuthal modes in a single ring configuration. In Bauerheim et al. (2014), this model was extended to a coupled set of one-dimensional networks for the analysis of two-ring configurations. The network approach is computationally efficient and allows for phenomenological interpretation of thermo-acoustic instabilities within the limits of moderately complex geometries.

In situations where more complex and detailed effects need to be accounted for, such as the response of a spatially distributed flame, the one-dimensional propagation models must be replaced by the multi-dimensional wave equation, often formulated as a Helmholtz problem. In a recent study by Mensah \& Moeck (2015), a thermo-acoustic model based on the Helmholtz equation was used to exploit the discrete rotational symmetry, common to most energy conversion devices, and to isolate special solutions of so-called Bloch type. These solutions are obtained by considering a single representative segment of the geometry, with the appropriate Bloch-type boundary conditions, creating an $N$-periodic geometry, significantly reducing the computational effort required to determine the eigenstructure of the full system. The Bloch-wave formalism supports the appropriate boundary conditions that account for the influence of adjacent periodic elements along the azimuthal direction whilst constructing all possible, globally periodic modes of the full system. Mathematically, this technique is closest to the framework employed in this article; however, non-modal characteristics and trans-unit behavior are more easily formulated and evaluated following the methodology presented in Schmid et al. (2017). In light of the inherently azimuthal and non-normal nature of the unstable modes of $N$-periodic systems (Balasubramanian \& Sujith 2008), this article focuses on the manner in which unsteady pressure in the chamber (azimuthal modes) can lead to unsteady flow rates within the burners, and thus couple the burner elements of an $N$ periodic energy conversion device into synchronized groups. A physical motivation for this study is given by the observation of azimuthal structures - especially rotating structures in annular combustion chamber geometries. In order to model this trans-unit interaction phenomenon we consider a system consisting of one-dimensional burner elements coupled via a one-dimensional chamber and plenum network. This network model is similar to the model presented in Bauerheim et al. (2014) and represents a convenient basis to analyze such coupled thermo-acoustic systems. We employ a roots-of-unity formalism (Schmid et al. 2017) that exploits the $N$-periodic nature of the configuration, similar to Mensah \& Moeck (2015), in order to efficiently compute the eigenstructure and non-modal response behavior of the global system.

While linear stability analysis is capable of predicting the onset of large-scale azimuthal thermo-acoustic oscillations as a function of system parameters (bifurcation point), it cannot predict the final amplitude of the bifurcated state of the system and the transition process, since it describes only the initial phase of the perturbation's evolution. On the other hand, non-normal or transient effects can lead to complex behaviors, such as triggering and bistability (Juniper 2011), only when the nonlinear nature of the system dictates that the bifurcation is subcritical (Sujith et al. 2016). Finally, nonlinear effects determine the nature of the final saturated state, it being a fixed point, a limit-cycle or a more complex solution, all observed experimentally in thermo-acoustic systems (Noiray et al. 2008; Kabiraj \& Sujith 2012; Jegadeesan \& Sujith 2013). Therefore, considering the impact of nonlinearities on the system is of fundamental importance and forms the second part of this paper.

Culick \& Kuentzmann (2006) used the method of averaging to derive the amplitude evolution for thermo-acoustic models with one or two oscillating modes and, within the same framework, Juniper (2012) described how the averaged quantities can be connected 
to the flame describing function methodology. Ghirardo et al. (2015) and Ghirardo et al. (2016) applied the method of averaging to azimuthal thermo-acoustic instabilities where only the most unstable linear pair of azimuthal modes of a single annular chamber was considered. Subramanian et al. (2013) used the method of multiple scales to derive a Stuart-Landau equation at third order in perturbation magnitude, describing the evolution of the oscillation amplitude in a Rijke tube. The Landau coefficients showed that the Hopf bifurcation was subcritical and the low-amplitude limit-cycles arising close to the Hopf point were unstable, in agreement with experimental studies. But in this latter study, and similarly in Juniper (2012), only a Galerkin mode of a single frequency was used to describe the dynamics. Such an analysis can be an approximation for some thermo-acoustic networks, however, considering a single or single pair of acoustic modes (with identical frequency) may alter the nature and amplitude of the oscillations, as was discussed by Ghirardo et al. (2016), Jahnke \& Culick (1994) and Ananthkrishnan et al. (2005). A weakly nonlinear analysis - by constructing an asymptotic expansion of the governing equations in the vicinity of the system's bifurcation point - can overcome limitations of the Galerkin approach and track the evolution of finite-amplitude oscillations. In other words, weakly nonlinear analysis reveals the mechanism behind finite amplitude thermo-acoustic oscillations inherent to annular combustors. This tool has been recently used to analyze thermo-acoustic instabilities of a ducted geometry (Orchini et al. 2016), while application to an annular problem poses a challenge on account of the system's discrete rotational symmetry (Ghirardo et al. 2016). This additional complexity is handled in the current study by the roots-of-unity method (Schmid et al. 2017) that, once again, exploits the block-circulant nature of the discrete dynamical system representation (Sayadi et al. 2014) of the system. Specifically, in case of the asymptotic expansion, the roots-of-unity method leverages the system's $N$-periodicity to transform the linear operator in a manner that simplifies the computation of its algebraic and geometric multiplicity. Hence, with the help of a nonlinear phenomenological expression for the heat release rate model, the roots-of-unity formalism and a uniformly valid asymptotic expansion of the discrete system's weakly nonlinear behavior, we are able to analyze the complete linear, non-normal and weakly nonlinear behavior of the plenumburner-chamber configuration in the vicinity of its Hopf-type bifurcation point, whilst retaining the contribution of multiple acoustic modes on the dynamics of the thermoacoustic system. This provides a comprehensive and global framework to investigate the nature of thermo-acoustic instabilities in annular combustion chambers.

This paper is organized as follows. In section 2 the equations governing the acoustic waves inside the one-dimensional burner and annulus are described, and a model for the heating source is introduced. Section 3 describes the spatial discretization and coupling between the burner and chamber/plenum sections of the model. The results of the linear modal stability of the linearized delay-differential system and the non-modal response analysis for a globally stable configuration are presented and analyzed in section 4 . Section 4.3 investigates the nature of the dynamical system, representing the nonlinear thermo-acoustic system close to its Hopf point by utilizing a uniformly valid weakly nonlinear asymptotic expansion of the limit-cycle oscillations. Lastly, a summary of our results and concluding remarks are offered in section 5 .

\section{Physical model and governing equations}

Azimuthal instabilities are a common feature in energy conversion devices with $\mathrm{N}$ periodic assemblies of identical units, such as gas turbines (Lieuwen \& Yang 2005; Poinsot \& Veynante 2005). In order to correctly capture and predict them, it is necessary to 


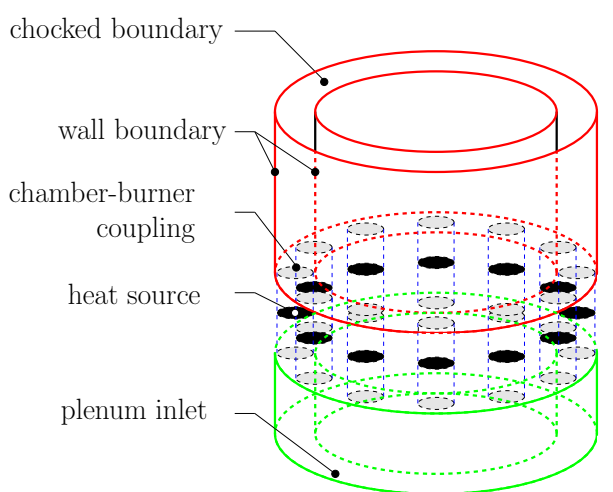

FiguRE 1. Sketch of an $N$-periodic, three-dimensional plenum-burner-chamber configuration consisting of two annular chambers linked by burners.

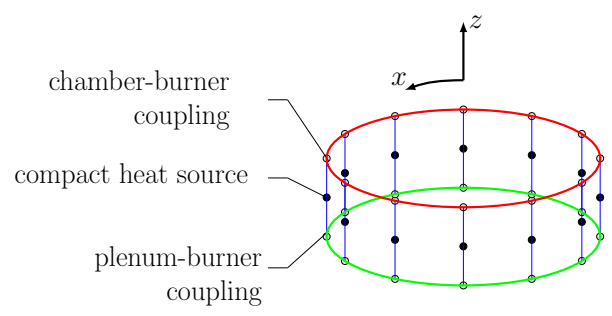

FIGURE 2. Sketch of an $N$-periodic one-dimensional plenum-burner-chamber configuration consisting of two one-dimensional ring-like chambers coupled together by one-dimensional burners. This simpler model captures the spatial dimensions along which the largest (compared to the compact heater) wavelength acoustic waves that manifest themselves inside the combustor.

devise a model that can account for the dynamics of a single burner as well as the coupling across elements. One-dimensional ducts with a compact heat source are widely used to describe single-burner geometries (Dowling 1995), and here we follow a similar approach. Our model is composed of $N$ identical units, assembled in a ring configuration, as shown in figure 1, where the annular geometry is displayed. This model is motivated by the geometrical configuration considered in Laera et al. (2017), which consists of $N=$ 12 identical matrix injectors with laminar flow and well-documented flame-describing functions.

The $N$-periodic configuration is further simplified by representing the threedimensional plenum and chamber as one-dimensional annuli and the burners as one-dimensional vertical ducts, each containing a compact heat source, as shown in figure 2. The compact heat source is modeled using a saturation-type nonlinear model that reduces to the $n$ - $\tau$-model in the linear limit (Orchini et al. 2016). The top and bottom boundaries of the burner open into the annular chamber and plenum elements of the combustion device. The annulus is modeled only along the periodic azimuthal direction, as illustrated in figure 2. The burner and chamber/plenum elements are coupled together using the jump relation obtained from the conservation of mass at the ends of the burner, where the elements are linked.

\subsection{Governing equations}

In what follows, we use $x$ as the azimuthal coordinate and $z$ as the longitudinal coordinate, as shown in figure 2. We consider the linear non-reacting one-dimensional acoustic equations,

$$
\left\{\begin{array}{l}
\rho_{0} \frac{\partial \tilde{u}}{\partial \tilde{t}}+\frac{\partial \tilde{p}}{\partial \tilde{x}}=0 \\
\frac{\partial \tilde{p}}{\partial \tilde{t}}+c_{0}^{2} \rho_{0} \frac{\partial \tilde{u}}{\partial \tilde{x}}=0
\end{array}\right.
$$

where the unperturbed base-flow variables, indicated by the subscript ${ }_{0}$, are $\rho_{0}, c_{0}, p_{0}$, $T_{0}$ and $u_{0} \ll c_{0}$, representing the density, speed of sound, pressure, temperature and velocity, respectively. These equations fully describe the propagation of one-dimensional acoustic waves in the absence of any heat source or dissipation. 
A non-dimensional form of the above equations is formulated by utilizing the meanflow properties of the system downstream of the heat source (indicated by the subscript $\left.{ }_{d}\right)$. The dimensional variables and coordinates can then be written, using the reference scales for velocity $\left(u_{0, d}\right)$, pressure $\left(\gamma p_{0} u_{0, d} / c_{0, d}\right)$, length $\left(L_{0}\right.$, the length of the burner) and time $\left(L_{0} / c_{0, d}\right)$, as

$$
\tilde{u}=u_{0, d} u, \quad \tilde{p}=\gamma p_{0} \frac{u_{0, d}}{c_{0, d}} p=\gamma p_{0} M p, \quad \tilde{x}=L_{0} x, \quad \tilde{t}=\left(L_{0} / c_{0, d}\right) t,
$$

where the quantities without a tilde or subscript 0 are dimensionless, and the mean pressure is defined as $p_{0}=p_{0, u}=p_{0, d}$ (where $p_{0, u}$ is the upstream mean value).

Substituting (2.2) into the dimensional governing equations (2.1) and making use of the definition of $c_{0, d}$, and the ideal gas law, yields the dimensionless governing equations

$$
\left\{\begin{array}{l}
\frac{\partial u}{\partial t}+\left(\frac{c_{0}}{c_{0, d}}\right)^{2} \frac{\partial p}{\partial x}=0 \\
\frac{\partial p}{\partial t}+\frac{\partial u}{\partial x}-\nu \frac{\partial^{2} p}{\partial x^{2}}=0
\end{array}\right.
$$

where, $c_{0}$ is a general representation of the speed of sound on either side of the flame. Equations (2.3) includes a term to model dissipative phenomena in the system, with non-dimensional viscosity denoted by $\nu$. This damping emulates acoustic losses, such as boundary layer losses, within the walls of the modeled annular combustor. The value of the non-dimensional number $\nu=0.047$ is chosen to approximate the damping model presented in Sayadi et al. (2014) over our frequency range of interest. In subsequent sections, $x_{c}, x_{p}$ and $z$ coordinates will be used to describe the one-dimensional perimeter of the chamber, plenum and the longitudinal direction along the burners, respectively.

\subsubsection{Acoustic equations inside the duct}

When a heat source is introduced into the system, the time rate of change of pressure is expressed in terms of the density and entropy as follows,

$$
\frac{\partial \tilde{p}}{\partial \tilde{t}}=c_{0}^{2} \frac{\partial \tilde{\rho}}{\partial \tilde{t}}+(\gamma-1) \tilde{\dot{Q}} \delta\left(\tilde{z}-\tilde{z}_{f}\right)
$$

where $\tilde{\dot{Q}}$ is the heat release perturbation per unit volume per unit time, given by $\tilde{\dot{Q}}=\rho_{0} T_{0} \frac{\partial \tilde{s}}{\partial \tilde{t}}(\tilde{s}$, representing entropy-per-unit-mass perturbations $), z_{f}$ is the location of the flame and $\delta(z)$ represents the dirac delta distribution along the $z$-axis. The above expression thus modifies the set of equations (2.3) as follows

$$
\left\{\begin{array}{l}
\frac{\partial w}{\partial t}+\left(\frac{c_{0}}{c_{0, d}}\right)^{2} \frac{\partial p}{\partial z}=0 \\
\frac{\partial p}{\partial t}+\frac{\partial w}{\partial z}-\nu \frac{\partial^{2} p}{\partial z^{2}}-\frac{(\gamma-1) L_{0}}{\gamma p_{0} w_{0, d}} \tilde{\dot{Q}} \delta\left(z-z_{f}\right)=0
\end{array}\right.
$$

where $w$ is the non-dimensional longitudinal velocity along the burners. The heat release model is then invoked to provide the following nonlinear representation of the heat-release perturbations per unit volume per unit time, in non-dimensional form, as 


$$
\frac{(\gamma-1) L_{0}}{\gamma p_{0} w_{0, d}} \tilde{\dot{Q}}=n f\left(w_{f, \tau}\right)
$$

where $f\left(w_{f, \tau}\right)=\alpha_{1} w_{f, \tau}+\alpha_{2} w_{f, \tau}^{2}+\alpha_{3} w_{f, \tau}^{3}+\alpha_{4} w_{f, \tau}^{4}+\alpha_{5} w_{f, \tau}^{5}$ and $n$ stands for the non-dimensional strength of the flame. The velocity $w_{f, \tau}$ is commonly taken to be the velocity value on the cold side of the heat source with a time delayed $(\tau)$ flame response; the coefficients $\alpha_{i}, i=1, \cdots, 5$, depend on the choice of the flame model. In this study we use the values

$$
\alpha_{1}=1, \quad \alpha_{2}=-0.33, \quad \alpha_{3}=0.0028, \alpha_{4}=0.1736, \quad \alpha_{5}=-0.0636,
$$

in order to approximate the saturation-type nonlinearity demonstrated by the King's law flame model. Section 4.3.5 discusses this flame model and the $\alpha_{i}$ in more detail.

\subsubsection{Coupling the chamber and the plenum to the duct}

While equations (2.5) are used to represent the flame dynamics and the propagation of the longitudinal acoustic perturbations within the burner subunits, they have yet to be linked in the azimuthal direction to the annular chamber and plenum. This is realized by coupling the modified wave equation (2.5) solved over the one-dimensional burner elements parallel to the axis of symmetry, with the dissipative wave equation (2.3) solved over the one-dimensional azimuthal direction perpendicular to the axis of symmetry, by ensuring the conservation of mass at the ends of the burner. This form of coupling was inspired by the work presented in Bauerheim et al. (2014). The final set of equations that results from this azimuthal coupling, along the coordinates of $x_{c}$ and $x_{p}$ that represent the perimeter of the chamber and plenum, respectively, is expressed as (refer Appendix A for details)

$$
\left\{\begin{array}{l}
\frac{\partial w}{\partial t}+\left(\frac{c_{0}}{c_{0, d}}\right)^{2} \frac{\partial p_{z}}{\partial z}=0 \\
\frac{\partial p_{z}}{\partial t}+\frac{\partial w}{\partial z}-\nu \frac{\partial^{2} p_{z}}{\partial z^{2}}-n f\left(w_{f, \tau}\right) \delta\left(z-z_{f}\right)=0
\end{array}\right.
$$

in the burner elements, and

$$
\left\{\begin{array}{l}
\frac{\partial u}{\partial t}+\left(\frac{c_{0}}{c_{0, d}}\right)^{2} \frac{\partial p}{\partial x}=0 \\
\frac{\partial p}{\partial t}+\frac{\partial u}{\partial x}-\nu \frac{\partial^{2} p}{\partial x^{2}}=0 \\
\frac{\partial p}{\partial t}+\left(\frac{\partial u}{\partial x_{c}}-\frac{w S_{b}}{\int S_{c} d x_{c}}\right)-\nu\left(\frac{\partial^{2} p}{\partial x_{c}^{2}}-\frac{\left.S_{i}\left(\partial p_{z} / \partial z\right)\right|_{z=1}}{\int S_{c} d x_{c}}\right)=0 \text { when } x_{c}=x_{b, c} \\
\frac{\partial p}{\partial t}+\left(\frac{\partial u}{\partial x_{p}}+\frac{w S_{b}}{\int S_{p} d x_{p}}\right)-\nu\left(\frac{\partial^{2} p}{\partial x_{p}^{2}}+\frac{\left.S_{i}\left(\partial p_{z} / \partial z\right)\right|_{z=0}}{\int S_{p} d x_{p}}\right)=0 \text { when } x_{p}=x_{b, p}
\end{array}\right.
$$

in the chamber. Here, $\left(p_{z}, w\right)$ and $(p, u)$ denote the pressure and axial velocity in the burner and the pressure and velocity along the perimeter of the chamber (or plenum), respectively. The coordinates $x_{b, c}$ ( or $x_{b, p}$ ) represent the location where a burner is coupled to the chamber ( or plenum). This model was discretized as per $\S 3$ and 

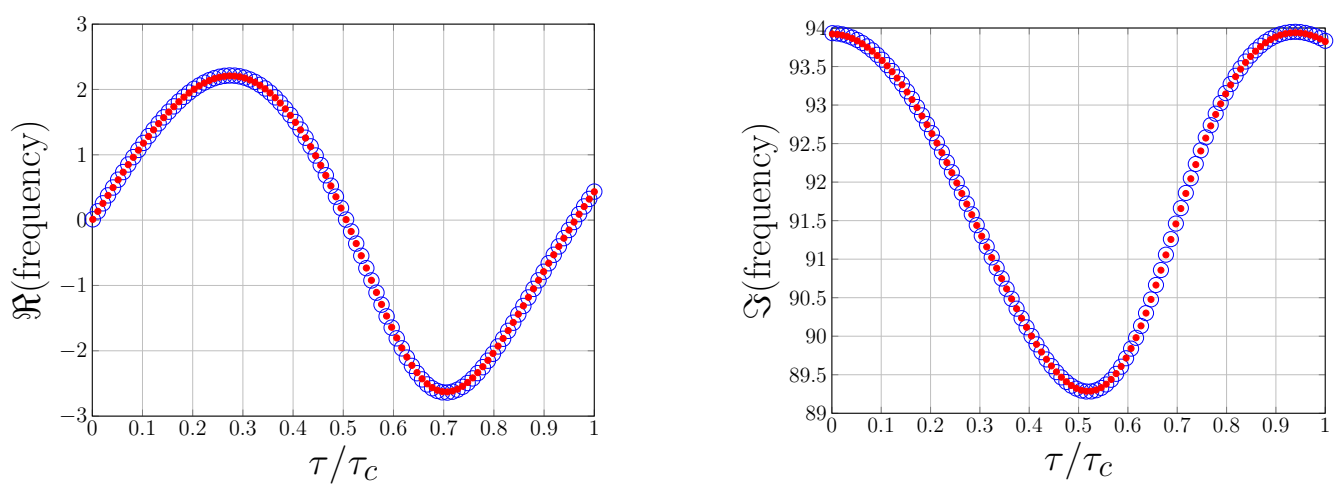

FiguRE 3. Real (left) and imaginary (right) part of the eigenfrequency corresponding to the first weakly coupled chamber mode. Red and blue circles represent the network model and discrete state space model results, respectively. $\tau / \tau_{c}$ represents the non-dimensional time delay associated with the flame (Bauerheim et al. 2014).

validated against the network model proposed in Bauerheim et al. (2014). The results of this validation are presented in figure 3, where the real and imaginary part of the eigenfrequency corresponding to the first weakly coupled chamber mode (WCC1; Bauerheim et al. (2014)) for an annular combustor with four burner elements, both from the network model and the discrete state space model, are successfully compared.

\subsection{Global dynamics from single-unit analysis}

The governing equations for the plenum burner and chamber configurations have to be formulated for an assembly of $N$ identical subunits. This formulation reveals a distinct structure of the governing system matrix: a block-circulant layout, where each identical block on the diagonal describes the motion in each unit, while the off-diagonal blocks account for the interaction with other units. In the analysis of the global system of $N$ units, this block-circulant structure can be exploited, and all computations can be performed on a single unit without forgoing the inter-unit and trans-unit dynamics. The mathematical transformation from the global system of equations to a single-unit formulation uses a roots-of-unity concept, which is akin to a Bloch-wave formalism; details can be found in Schmid et al. (2017).

Any desired quantity of the full system - either modal or non-modal - can then be formulated on a single-unit system and computed while varying the root-of-unity. Here, the root-of-unity index $(j)$ refers to a form of boundary condition specifying the phase difference $(2 \pi j / N)$ across a single sector, of a solution, in the azimuthal direction. In addition, the mutual influence of distinct units as well as the trans-unit dynamics can be described and quantified by considering only a sequence of single-unit systems. As a consequence, the computational cost (e.g., for a modal analysis) is reduced from $\mathcal{O}\left(N^{3} h^{3}\right)$ for the full global analysis to $\mathcal{O}\left(N h^{3}\right)$ where $h$ represents the number of degrees of freedom in a single unit; a savings factor of $N^{2}$ can be realized for our case of $N=12$ units, which is a savings of two orders of magnitude.

It is important to note that non-modal effects can only arise from the non-normality of the single-unit dynamics. The linking of identical units does not produce additional amplification potential. Nonetheless, the response of the global system to external forcing, for example, can depend crucially on the interaction across subunits, and the response for one root-of-unity solution can significantly exceed the response for another solution (see 


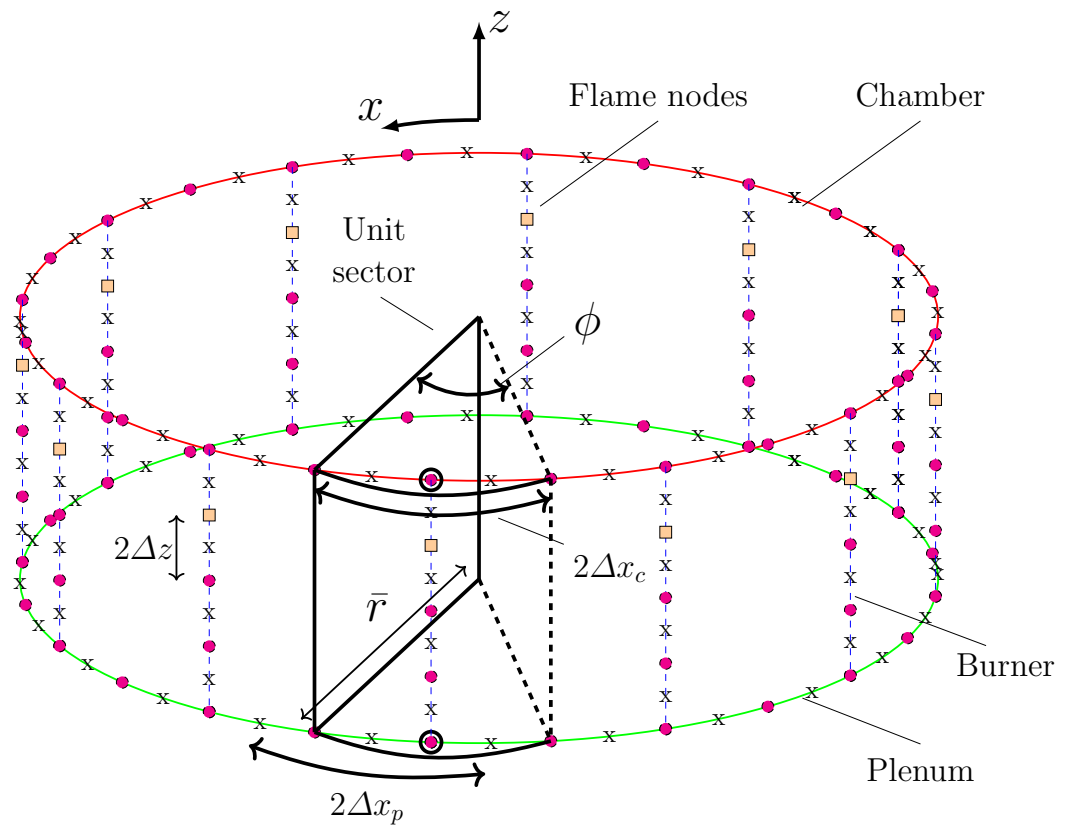

FiguRE 4. Staggered grid formation of pressures (circles) and velocities ( $\mathrm{x}$ ) within the one-dimensional plenum-burner-chamber geometry. The angle subtended and the mean radius of the unit sector are labeled as $\phi=2 \pi / N$ and $\bar{r}$, respectively. The pressure nodes at which the burner is coupled to the chamber and plenum are highlighted. The pressure and velocity nodes within the unit sector (except those on the dashed lines) constitute that sector's state variables.

figure 7). In fact, the type of response for varying forcing frequencies can favor different periodicities and switch discontinuously as the frequency is modified.

\section{Numerical framework}

This section describes the proposed discrete state space model, including the numerical treatment of the spatial operators, the coupling between the plenum, burner and chamber components of the combustor, and the time delay inherent to the nonlinear flame model present in the set of equations (2.8) and (2.9).

\subsection{Discretized governing equations: burner}

A staggered arrangement of the variables is used to discretize the one-dimensional burner geometry (see figure 4). The boundary conditions at the first and last pressure nodes of the burner, located at $z=0$ and $z=1$ accordingly, are inferred by the coupling with the plenum and the chamber. The numerical framework of Sayadi et al. (2014) is adopted here, where the spatial derivatives along the burner are represented using a second-order central difference scheme. A ghost fluid treatment (Fedkiw et al. 1999) for the velocity discontinuity across the heat source is employed to retain the compact character of the acoustic source, formally reducing the order of accuracy to first order in the vicinity of the singularity. In this formalism, the heat source coincides with a pressure node. The spatial discretization is completed by the approximation of the damping, as described in section 2.1, and is presented in the following for a unit-sector.

The state vector corresponding to the burner, containing $M_{b}$ pressure nodes and $M_{b}+1$ 
velocity nodes, is given by

$$
\boldsymbol{x}_{b}=\left[w_{b}^{1}, p_{b}^{1}, w_{b}^{2}, p_{b}^{2}, \cdots, p_{b}^{M_{b}}, w_{b}^{M_{b}+1}\right]^{\mathrm{T}} .
$$

Discretization of governing equations (2.3) takes the following form

$$
\begin{aligned}
\frac{d w_{b}^{i}}{d t} & =-\left(\frac{c_{0}}{c_{0, d}}\right)^{2}\left(\frac{p_{b}^{i}-p_{b}^{i-1}}{2 \Delta z}\right), \\
\frac{d p_{b}^{i}}{d t} & =-\left(\frac{w_{b}^{i+1}-w_{b}^{i}}{2 \Delta z}\right)+\nu\left(\frac{p_{b}^{i+1}-2 p_{b}^{i}+p_{b}^{i-1}}{4 \Delta z^{2}}\right) .
\end{aligned}
$$

The pressure nodes at the ends of the burner are shared with the plenum and chamber units. The temporal evolution of pressure at these shared nodes is governed by their respective mass flux and viscous dissipation, influenced both by the burner and annular chamber/plenum. The discrete equation governing the rate of change of pressure at these points is discussed in section 3.3.

A discrete dynamical system representation of the stand-alone burner equations (2.8) can then be represented by

$$
\dot{\boldsymbol{x}}_{b}=\boldsymbol{A}_{b} \boldsymbol{x}_{b}+\boldsymbol{A}_{b c} \boldsymbol{x}_{c}+\boldsymbol{A}_{b p} \boldsymbol{x}_{p}+n f\left(w_{b}^{i_{f}}(t-\tau)\right) \hat{\boldsymbol{e}}_{\left(i_{f}-1\right)},
$$

where, $\hat{\boldsymbol{e}}_{i}$ is the unit vector with a unit entry at the $i^{\text {th }}$ index, $\boldsymbol{x}_{c}$ and $\boldsymbol{x}_{p}$ are the state vectors corresponding to the chamber and plenum sections, respectively, and will be defined in the next section, $i_{f} \Delta z=z_{f}\left(\Delta z\right.$ is shown in figure 4), matrix $\boldsymbol{A}_{b}$ is constructed using equation set (3.2) and matrices $\boldsymbol{A}_{b c}$ and $\boldsymbol{A}_{b p}$ represent the burner-chamber and burner-plenum coupling, respectively. Implementation of the delayed term $w_{b}^{i_{b}}(t-\tau)$ is explained in $\S 3.4$.

\subsection{Discretized governing equations: chamber/plenum}

A staggered arrangement of the variables on a one-dimensional axisymmetric structured mesh (see figure 4) is also used to discretize the chamber/plenum geometry. The azimuthal boundary conditions are specified according to the roots-of-unity formalism for single-sector (unit-sector) computations. The spatial derivatives across the chamber are represented using a second-order central difference approximation.

In what follows subscript $\xi$ represents either chamber $(c)$ or plenum $\left({ }_{p}\right)$ specific quantities. The resulting unit-sector state vector corresponding to the chamber/plenum, containing $M_{\xi}$ pressure nodes, and an equal number of velocity nodes, is given by

$$
\boldsymbol{x}_{\xi}=\left[p_{\xi}^{1}, u_{\xi}^{1}, p_{\xi}^{2}, u_{\xi}^{2}, \cdots, p_{\xi}^{M_{\xi}}, u_{\xi}^{M_{\xi}}\right]^{\mathrm{T}} .
$$

Discretization of governing equations (2.3) takes the following form

$$
\begin{aligned}
\frac{d u_{\xi}^{i}}{d t} & =-\left(\frac{c_{0}}{c_{0, d}}\right)^{2}\left(\frac{p_{\xi}^{i+1}-p_{\xi}^{i}}{2 \Delta x_{\xi}}\right), \\
\frac{d p_{\xi}^{i}}{d t} & =-\left(\frac{u_{\xi}^{i}-u_{\xi}^{i-1}}{2 \Delta x_{\xi}}\right)+\nu\left(\frac{p_{\xi}^{i+1}-2 p_{\xi}^{i}+p_{\xi}^{i-1}}{4 \Delta x_{\xi}^{2}}\right) .
\end{aligned}
$$

The above equations are valid if $i \in\left\{1, M_{\xi}\right\}$, else the roots-of-unity method imposes a boundary condition of the form $u_{\xi}^{0}=u_{\xi}^{M_{\xi}} / \rho_{j}$ and $p_{\xi}^{0}=p_{\xi}^{M_{\xi}} / \rho_{j}$ when $i=1$, and $p_{\xi}^{M_{\xi}+1}=p_{\xi}^{1} \rho_{j}$ when $i=M_{\xi}$, where $\rho_{j}=\exp (\mathbf{i} 2 \pi j / N)$. 
A discrete dynamical system representation of the stand-alone chamber/plenum equations (2.9) can then be represented by

$$
\begin{aligned}
\dot{\boldsymbol{x}}_{\xi} & =\left\{\boldsymbol{A}_{\xi}^{\prime}(j)-\nu\left(S_{b} /\left(S_{\xi} 4 \Delta x_{\xi} \Delta z\right)\right) \hat{\boldsymbol{e}}_{i_{\xi}} \otimes \hat{\boldsymbol{e}}_{i_{\xi}}^{\mathrm{T}}\right\} \boldsymbol{x}_{\xi}+\boldsymbol{A}_{\xi b} \boldsymbol{x}_{b}, \\
& =\boldsymbol{A}_{\xi}(j) \boldsymbol{x}_{\xi}+\boldsymbol{A}_{\xi b} \boldsymbol{x}_{b},
\end{aligned}
$$

where, matrix $\boldsymbol{A}_{\xi}^{\prime}$ is constructed using equation set (3.5), matrix $\boldsymbol{A}_{\xi b}$ represents the additional terms, mentioned in equations (2.9), that describe the influence of the burner on the shared pressure nodes within each cavity and, $i_{\xi}$ is the index of the coupled pressure node (highlighted in figure 4 with dark circles), within each cavity and at the ends of a burner, in vector $\boldsymbol{x}_{\xi}$.

\subsection{Discretized governing equations: combined plenum-burner-chamber equations}

The structure of the nonlinear dynamical system, representing equations (2.8) and (2.9) within a unit-sector (shown in figure 4), is now given by

$$
\dot{\boldsymbol{x}}_{u s}=\left[\begin{array}{ccc}
\boldsymbol{A}_{c}(j) & \boldsymbol{A}_{c b} & 0 \\
\boldsymbol{A}_{b c} & \boldsymbol{A}_{b} & \boldsymbol{A}_{b p} \\
0 & \boldsymbol{A}_{p b} & \boldsymbol{A}_{p}(j)
\end{array}\right] \boldsymbol{x}_{u s}+\left[\begin{array}{c}
0 \\
n f\left(w_{b}^{i_{f}}(t-\tau)\right) \hat{\boldsymbol{e}}_{i_{f}} \\
0
\end{array}\right],
$$

where, $\boldsymbol{x}_{u s}=\left[\boldsymbol{x}_{c}^{\mathrm{T}}, \boldsymbol{x}_{b}^{\mathrm{T}}, \boldsymbol{x}_{p}^{\mathrm{T}}\right]^{\mathrm{T}}$, matrices of the form $\boldsymbol{A}_{b \xi}$ and $\boldsymbol{A}_{\xi b}$ are given by

$$
\begin{aligned}
& \boldsymbol{A}_{b c}=-\left(c_{0}^{2} /\left(c_{0, d}^{2} 2 \Delta z\right)\right) \hat{\boldsymbol{e}}_{2 M_{b}+1} \otimes \hat{\boldsymbol{e}}_{i_{c}}^{\mathrm{T}} \\
& \boldsymbol{A}_{b p}=\left(c_{0}^{2} /\left(c_{0, d}^{2} 2 \Delta z\right)\right) \hat{\boldsymbol{e}}_{1} \otimes \hat{\boldsymbol{e}}_{i_{p}}^{\mathrm{T}} \\
& \boldsymbol{A}_{c b}=\left(S_{b} /\left(S_{c} 2 \Delta x_{c}\right)\right) \hat{\boldsymbol{e}}_{i_{c}} \otimes \hat{\boldsymbol{e}}_{2 M_{b}+1}^{\mathrm{T}}+\nu\left\{\left(S_{b} /\left(S_{c} 4 \Delta x_{c} \Delta z\right)\right) \hat{\boldsymbol{e}}_{i_{c}} \otimes \hat{\boldsymbol{e}}_{2 M_{b}}^{\mathrm{T}}\right\} \\
& \boldsymbol{A}_{p b}=-\left(S_{b} /\left(S_{p} 2 \Delta x_{p}\right)\right) \hat{\boldsymbol{e}}_{i_{p}} \otimes \hat{\boldsymbol{e}}_{1}^{\mathrm{T}}+\nu\left\{\left(S_{b} /\left(S_{p} 4 \Delta x_{p} \Delta z\right)\right) \hat{\boldsymbol{e}}_{i_{p}} \otimes \hat{\boldsymbol{e}}_{2}^{\mathrm{T}}\right\}
\end{aligned}
$$

\subsection{Spectral and bifurcation analysis}

The discretization and subsequent linearization of equations (2.8) and (2.9) yield a set of linear delay-differential equations (DDE's). The stability properties of such systems can be analyzed using a technique proposed by Jarlebring (2008), which recasts the original DDE as a partial differential equation by introducing an additional independent variable in the form of a space-like memory dimension.

The analysis is performed on the discretized system of equations, described in sections $\S 3.1$ through $\S 3.3$, with the linearized version of the source term from the flame model given in equation (2.6). The parameters of interest in the ensuing spectral and bifurcation analysis are the non-dimensional flame strength $n$, its location $z_{f}\left(i_{f}\right)$, and the delay time $\tau$. The roots-of-unity formalism yields $N$ decoupled and linear semi-discrete delay differential equations for each $j \in\{0, \ldots, N-1\}$, cast in matrix form according to

$$
\dot{\boldsymbol{x}}_{u s}=\underbrace{\left[\begin{array}{ccc}
\boldsymbol{A}_{c}(j) & \boldsymbol{A}_{c b} & 0 \\
\boldsymbol{A}_{b c} & \boldsymbol{A}_{b} & \boldsymbol{A}_{b p} \\
0 & \boldsymbol{A}_{p b} & \boldsymbol{A}_{p}(j)
\end{array}\right]}_{\boldsymbol{A}_{0}(j)} \boldsymbol{x}_{u s}+\underbrace{\left[\begin{array}{ccc}
0 & 0 & 0 \\
0 & n \boldsymbol{A}_{b} \hat{\boldsymbol{e}}_{\left(i_{f}-1\right)}^{\mathrm{T}} & 0 \\
0 & 0 & 0
\end{array}\right]}_{\boldsymbol{A}_{1}} \boldsymbol{x}_{u s}(t-\tau),
$$

The matrix $\boldsymbol{A}_{0}(j)$ contains differentiation and damping operators together with the $j$-th root-of-unity azimuthal boundary condition, and $\boldsymbol{A}_{1}$ includes the linearized heat source. 
The eigenvectors and eigenvalues of this matrix satisfy the following relation (Jarlebring 2008)

$$
\left(\boldsymbol{A}_{0}(j)+\boldsymbol{A}_{1} e^{-\tau \lambda}\right) \boldsymbol{v}=\lambda \boldsymbol{v},
$$

where $\boldsymbol{v}$ represents an eigenvector and $\lambda$ the associated eigenvalue. Equation (3.10) constitutes a nonlinear eigenvalue problem that can be solved numerically using the PDE-method (Jarlebring 2008), The numerical techniques reported in Jarlebring (2008) are available in the software package DDE-BIFTOOL (Engelborghs et al. 2000), which is employed in this article to obtain neutral curves, corresponding to the different rootof-unity, against the parameters $n$ and $\tau$, for a given geometry, coupling parameters and flame location.

\section{Results and discussions}

In this section, the discrete model will be applied to the plenum-burner-chamber (PBC) configuration described in $\S 2$, focusing on the influence of model parameters such as flame strength and delay on the linear stability of the PBC system. The non-modal behavior is then investigated through a receptivity analysis to harmonic forcing and, finally, its weakly nonlinear behavior is assessed using an asymptotic expansion of the dynamical system represented by the governing equations (2.8) and (2.9). The linear and non-modal analyses provide insight into the short-term response to broadband environmental noise or to a specific excitation by components upstream or downstream of the combustion unit, whilst the manner in which such disturbances can pulse the system from a stable baseflow to sustained oscillations is quantified by a weakly nonlinear asymptotic expansion of the system's behavior. This uniformly valid asymptotic expansion reveals the subcritical nature of the nonlinearities inherent to the PBC configuration, by introducing multiple time scales to formally separate the fast and the slow dynamics. The fast timescale and the bifurcation point can be obtained from a linear stability analysis, on the other hand, the nonlinear evolution of the system close to this critical point can be described by a complex amplitude that is a function of the slow timescales. This amplitude evolution equation is given in the form of an ordinary differential equation - appearing as a Stuart-Landau equation - for every linearly unstable mode. Solving the latter equation is noticeably faster than time-marching the full nonlinear system and provides insight into the nonlinear interactions between the modes.

The twelve-burner geometrical configuration of the PBC system that we investigate has a heat source within each burner element (of length $L_{0}=0.0508 \mathrm{~m}$ ) at a distance $z_{f}=$ $0.88 L_{0}$ from the boundary it shares with the plenum. The burners themselves are coupled, in an $N$-periodic fashion, along the perimeter of the chamber and plenum of the PBC system. The inner radius, outer radius and height of the chamber are taken to be $0.24 \mathrm{~m}$, $0.197 \mathrm{~m}$ and $0.6 \mathrm{~m}$, respectively. Similarly, the inner radius, outer radius and height of the plenum are taken to be $0.24425 \mathrm{~m}, 0.19275 \mathrm{~m}$ and $0.1 \mathrm{~m}$, respectively. These dimensions are similar to the annular test case considered in Laera et al. (2017), but were modified by reducing the diameter and height of the plenum as well as increasing the height of the chamber to cause the first Hopf bifurcation to appear for a degenerate weakly coupled chamber mode (Bauerheim et al. 2014). This specific coupling between the components of the PBC configuration also ensures that the mode associated with the system's Hopf bifurcation is the lowest-frequency, globally periodic, $(j=1)$ azimuthal eigenmode (figure $5 \mathrm{~b})$ belonging to an eigenspace of dimension two; this particular degeneracy is critical in describing the nonlinear behavior of the system. 


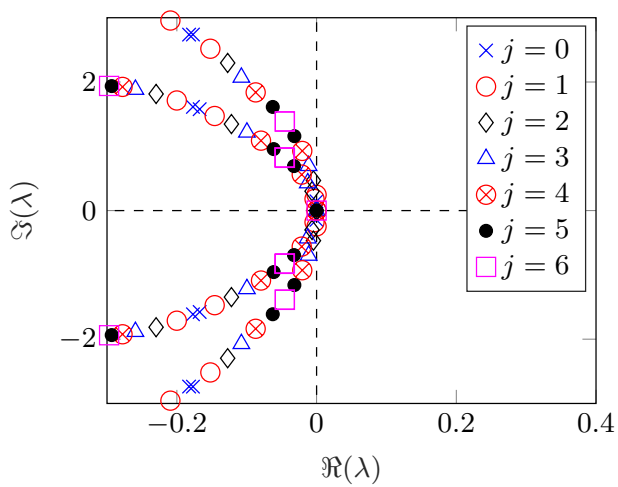

(a)

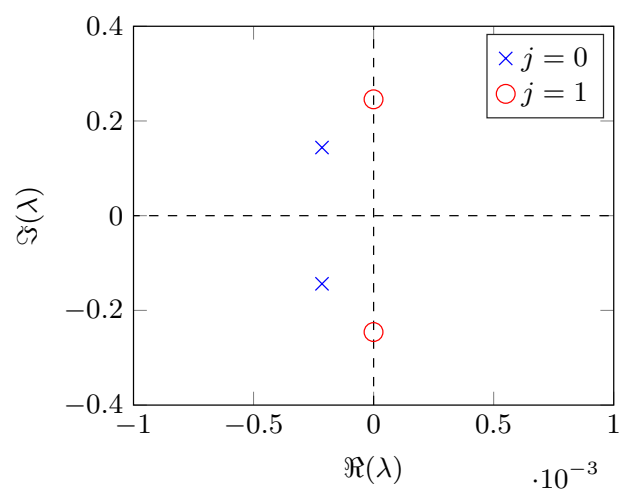

(b)

Figure 5. Eigenspectra of the PBC configuration for (a) $n=0.2425$ and $\tau=3.85$, and (b) close-up of the eigenspectrum in (a).

\subsection{Modal analysis}

We start by assessing the modal stability of thermo-acoustic waves in the PBC system based on a spectral analysis. Modal shapes and neutral curves of the configuration are also computed in order to shed more light on the systems linear stability. The spectrum, for non-dimensional time delay $\tau=3.85$ and flame strength $n=0.2425$, is displayed in figures 5(a) and 5(b). These parameters mark the onset of instabilities in the system, when the neutral curve corresponding to the root-of-unity $j=1$ shows a transition in linear stability and alters the dynamical behavior of the $N$-periodic system. Since, in this example, the $N$-periodic system consists of 12 units, 12 solutions of the roots-ofunity algorithm exist. However, roots for $j$ and $N-j$ (where $j \in\{1, \ldots, N-1\}$ ) yield the same eigenspectrum, stemming from degeneracy of dimension two. This degeneracy, while responsible for rich nonlinear thermo-acoustic behavior in annular combustion chambers (Ghirardo et al. 2016), does not affect the modal behavior of the system and hence, in all spectral and bifurcation plots, only a subset of the roots-of-unity with $j=0,1, \ldots, 6$ are considered.

The spatial structure of the eigenmode on the brink of a Hopf bifurcation, corresponding to the root-of-unity $j=1$, is visualized in figures $6(\mathrm{a}, \mathrm{b}, \mathrm{c})$. Figure $6(\mathrm{a})$ illustrates the structure of the mode along the perimeter of the annular chamber, figure $6(\mathrm{~b})$ presents the modal shape along the perimeter of the annular plenum, and figure $6(\mathrm{c})$ presents the variation in the burner. This eigenmode corresponds to a weakly coupled chamber (WCC) mode, based on the nomenclature used in Bauerheim et al. (2014), and corresponds to the lowest-frequency globally periodic azimuthal eigenmode of the system (see figure $5 \mathrm{~b}$ ) that also belongs to an eigenspace of dimension two $(j=1$ and $j=N-1)$.

The neutral curves of this PBC system, as a function of the non-dimensional strength $n$ and time delay $\tau$, for a fixed flame location are shown in figure 7. Each neutral curve in figure 7 represents the demarcation line across which an eigenmode, corresponding to a particular root-of-unit $j$, will change from stable to unstable behavior in the $(n, \tau)$ parameter space. Together, the neutral curves corresponding to $j=0$ and $j=1$ describe the positions of transition in linear stability, in the $n-\tau$ parameter space, for the system under consideration. The roots-of-unity corresponding to $j>2$ do not undergo a Hopf bifurcation in this portion of the $n-\tau$ space and are therefore not shown in the figure. The global stability cannot be fully captured when the analysis is restricted to unitperiodic eigenmodes $(j=0)$, as is evident from the more unstable behavior of eigenmodes 


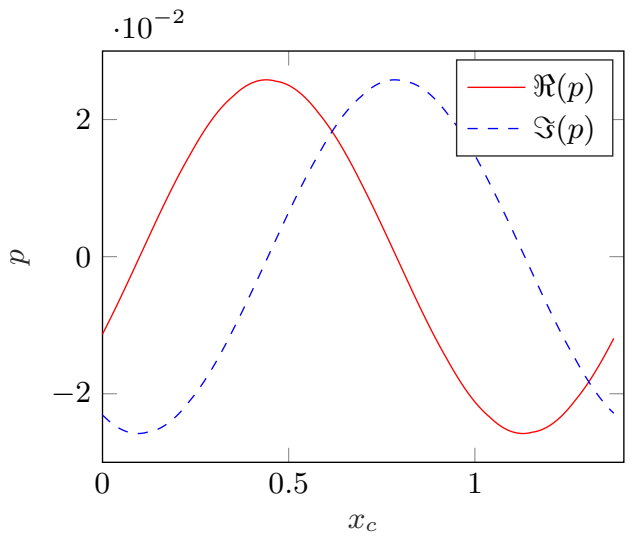

(a)



(b)

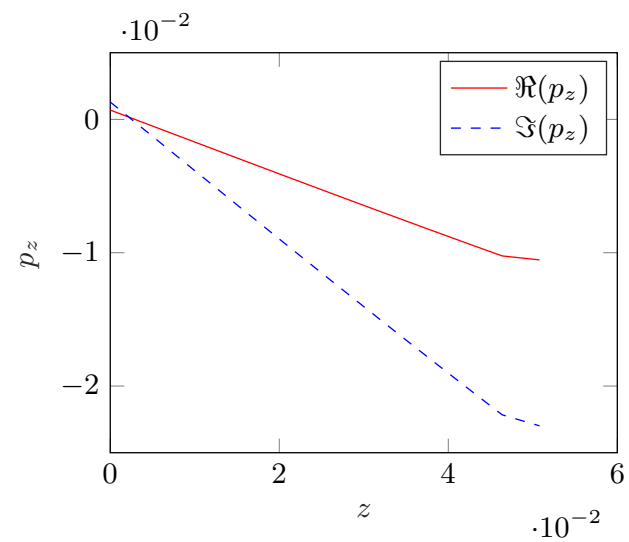

(c)

FIGURE 6. Structure of the eigenmode corresponding to the eigenvalue on the brink of undergoing a Hopf bifurcation, along the (a) chamber (b) plenum and (c) burner.

corresponding to the root-of-unity $j=1$. Furthermore, this figure shows that stability limits according to individual neutral curves vary in relation to one another over the $(n, \tau)$ parameter space, leading to variations in modal structure (eg. root-of-unity index $j$ ) of the most unstable eigenmode as a function of the system parameters.

\subsection{Non-modal analysis}

While combustion chambers can exhibit thermo-acoustic instabilities due to a positive feedback between flow, combustion and acoustic processes, they can also act as amplifiers of particular frequencies imposed on them by the upstream compressor and/or the downstream turbine stage in the stable regime. Assessing the response of $N$-periodic combustion systems to harmonic forcing is therefore important. Accordingly, this section is dedicated to the receptivity of the PBC system subject to harmonic forcing.

The system's response to small-amplitude harmonic forcing, with asymptotically stable eigenmodes, is measured by the maximum response-to-forcing ratio of the corresponding long-term solution (Schmid \& Henningson 2001), which is given by $\max _{\boldsymbol{x}_{f}} \frac{\left\|\boldsymbol{M}_{j} \boldsymbol{x}_{f}\right\|}{\left\|\boldsymbol{x}_{f}\right\|}$, where $\boldsymbol{M}_{j}=\left(\boldsymbol{A}_{\tau}(j)-\mathrm{i} \omega \boldsymbol{I}\right)^{-1} \boldsymbol{W}$ is the resolvent operator, and $\boldsymbol{x}_{f}$ is the spatial component of the 


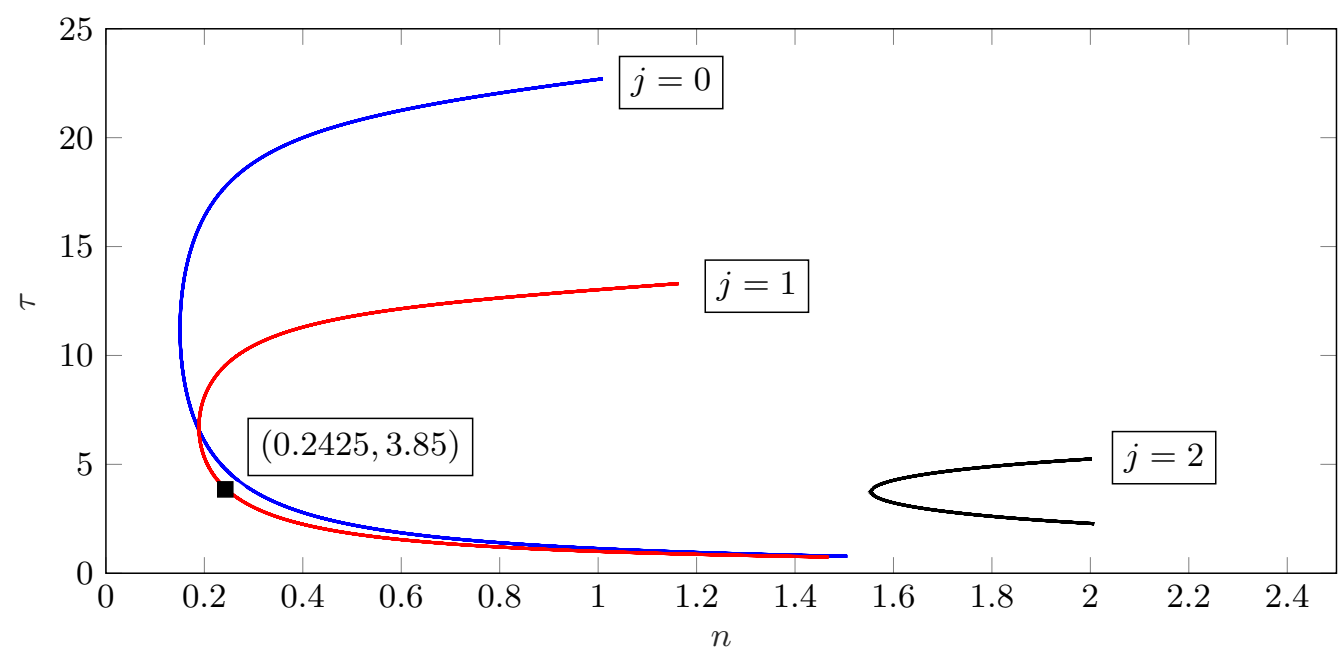

FiguRE 7. Neutral curve presented in the $n-\tau$ space for the PBC configuration. The black square represents the point in parameter space where the system will be studied. Here, the value of $z_{f}=0.88$.

forcing function. This expression defines the resolvent norm as the maximum value of this ratio. Matrix $\boldsymbol{A}_{\tau}(j)$ denotes the linear operator resulting from matrices $\boldsymbol{A}_{0}(j)$ and $\boldsymbol{A}_{1}$ in equation (3.9) after applying the PDE-method according to Jarlebring (2008), where subscript $j$ represents the specified root-of-unity index. An artifact of this PDE-method is that $\boldsymbol{x}_{f}$ represents the solution over the interval $[t-\tau, t]$. In order to avoid alteration of past values, the forcing is applied at the current instant only by means of a mask matrix $\boldsymbol{W}$. While the resolvent norm quantifies the largest gain, between the input forcing and the output response, the associated principal right and left singular vectors of $\boldsymbol{M}_{j}$ provide the spatial structure of the optimal forcing and response, respectively.

Using the resolvent norm, the receptivity of the PBC configuration is depicted in figure 8 , for every root-of-unity index. The figure illustrates that the global non-normal response of this system is the union of the most responsive behavior across all frequencies and rootof-unity indices. To highlight the non-normal nature of the system a reference normal response is plotted in black, computed as the reciprocal of the minimum distance between the imposed harmonic forcing frequency and the nearest eigenvalue of the linearized dynamical system. Comparing the global non-normal response of the system to the reference normal response shows that the largest departure from the normal response is observed for frequencies $\tilde{\omega} /(2 \pi)<1500 \mathrm{~Hz}$. Figure 9 (a) compares the modal and nonmodal responses for the $j=1$ root-of-unity index. This root-of-unity is the most unstable mode for the chosen values of $(n, \tau)$, as shown by figure 7 , making the analysis of its nonmodal behavior significant. Figure 9 shows two peaks at frequencies $\tilde{\omega} /(2 \pi)=709.9 \mathrm{~Hz}$, and $\tilde{\omega} /(2 \pi)=967.2 \mathrm{~Hz}$, which is expected, since these frequencies correspond to the eigenmodes of the modal system. However, when measured by the ratio of the resolvent response to normal response, shown in figure $9(\mathrm{~b})$, indicating the largest departure from normal behavior, the maximum value appears for $\tilde{\omega} /(2 \pi)=838.1 \mathrm{~Hz}$. This shows that the low frequency azimuthal modes of the PBC system exhibit a strong non-normal response to harmonic excitation even at frequencies far from the eigen-frequencies of the system. The corresponding left and right singular vectors of the resolvent operator $\boldsymbol{M}_{j}$, signifying the spatial shapes of the forcing and response functions at $\tilde{\omega} /(2 \pi)=838.1$ $\mathrm{Hz}$, is illustrated in figure 10. The shape of the response is similar to the eigenmode of 


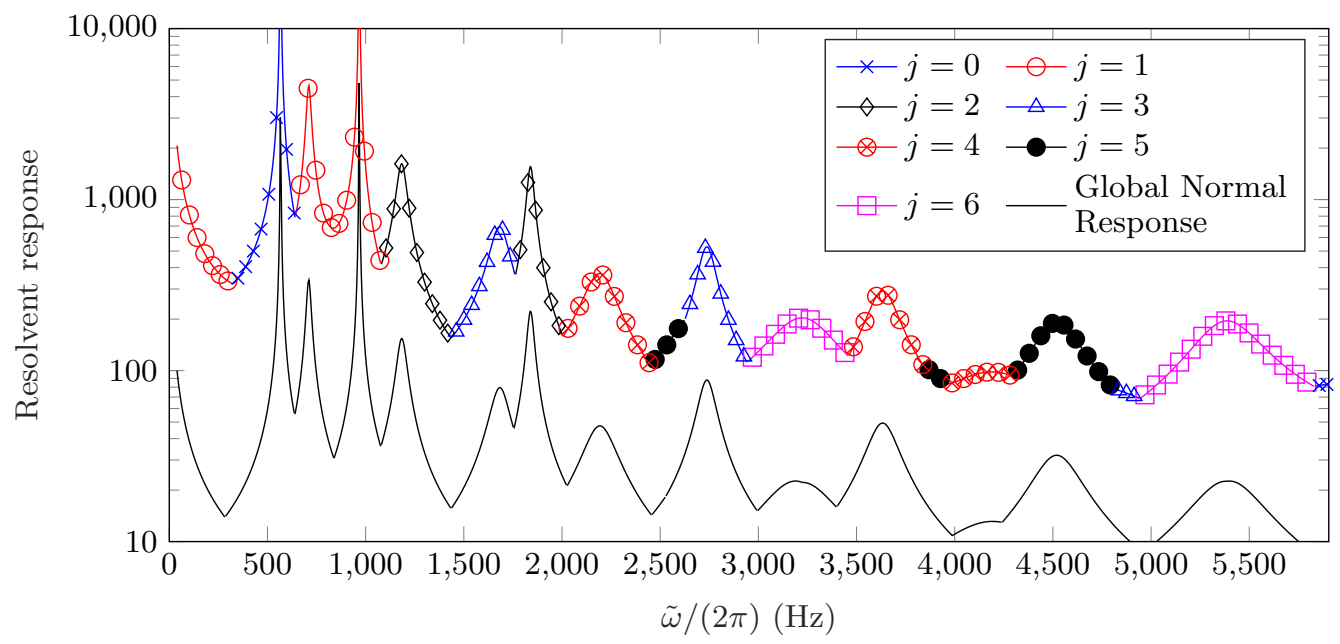

Figure 8. Non-modal response of the PBC configuration for $n=0.2425$ and $\tau=3.85$, and comparison to a normal solution.

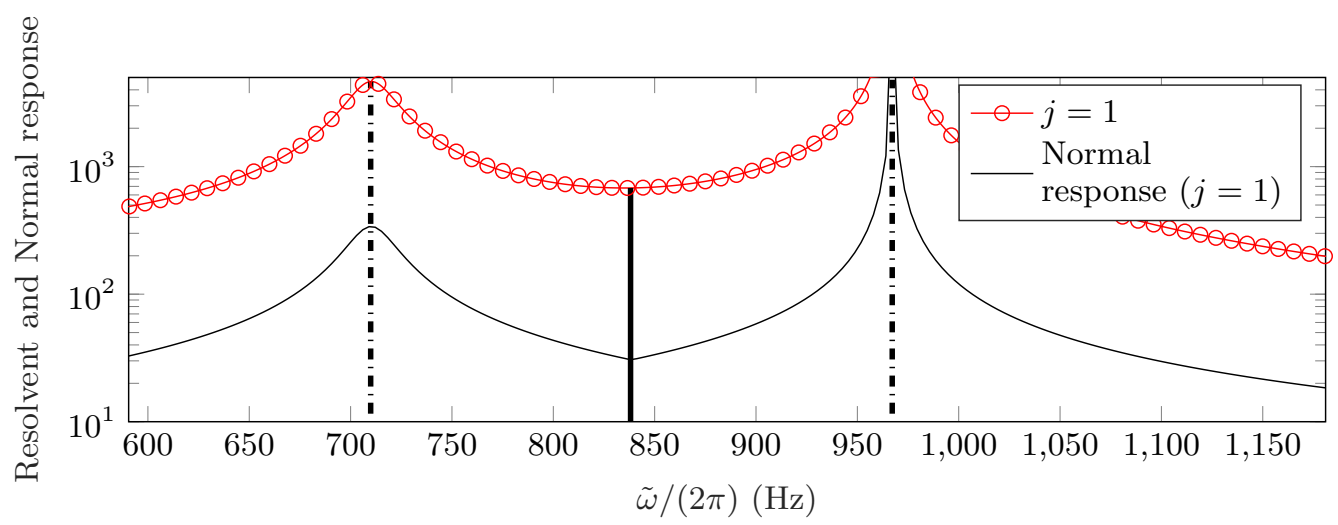

(a)

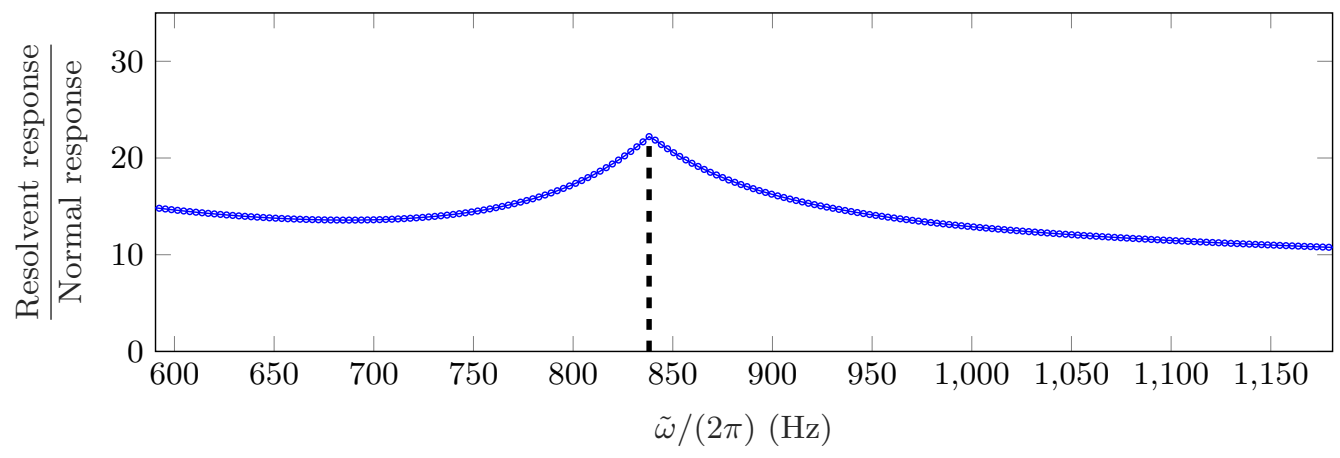

(b)

Figure 9. (a) Non-modal (red) and modal (black) response of the $j=1$ root-of-unity. (b) ratio of the resolvent response to the normal response as a function of frequency $(\tilde{\omega} /(2 \pi))$ 


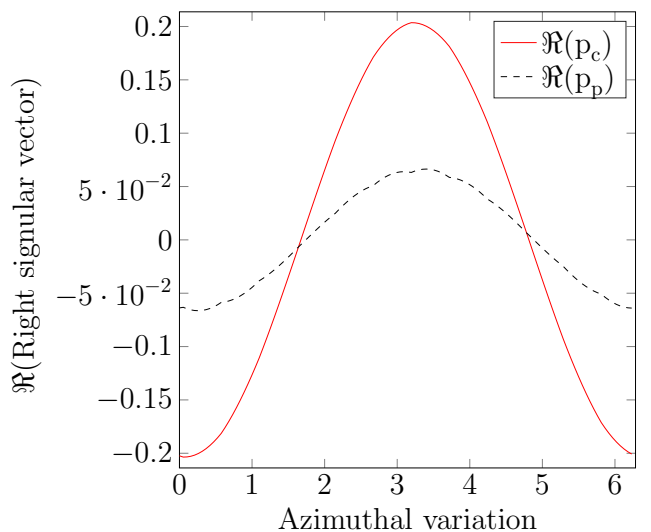

(a)

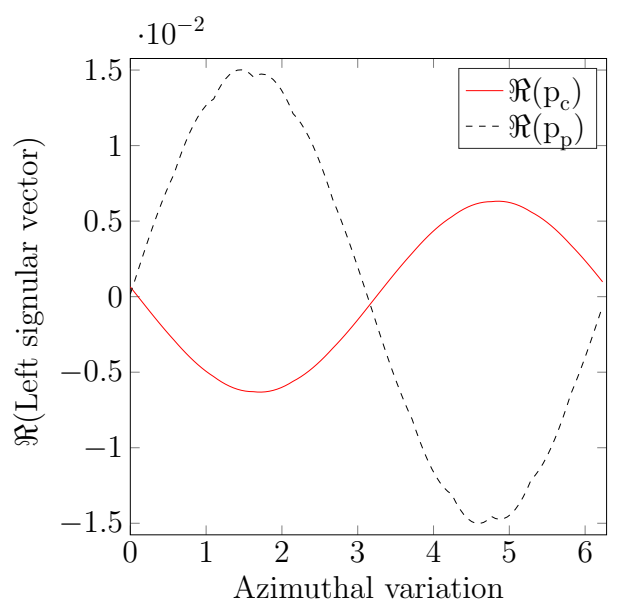

(c)

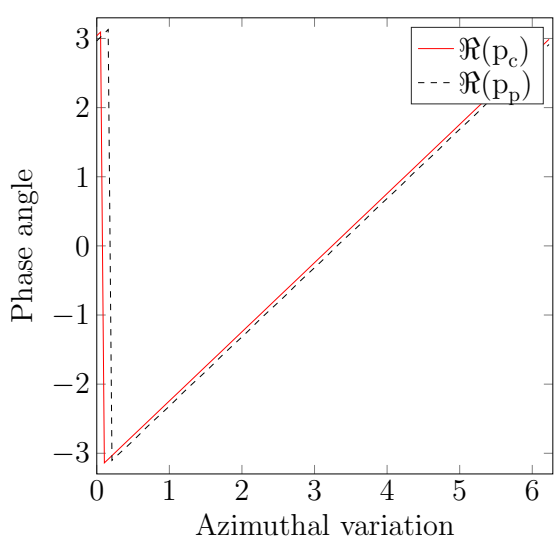

(b)

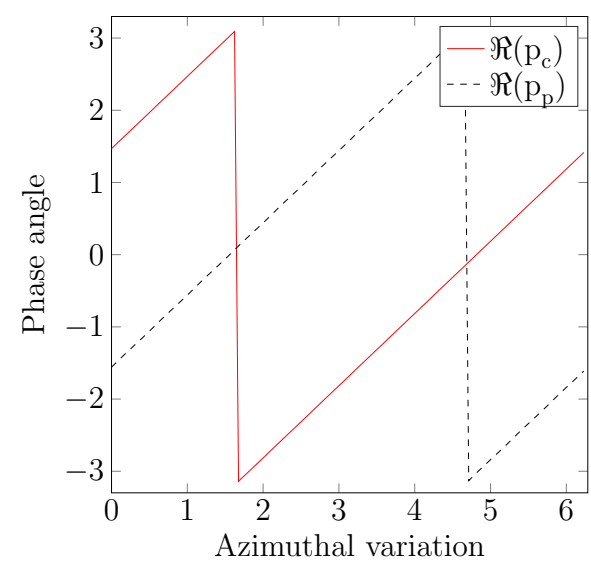

(d)

FiguRE 10. The real part and the phase of the right and left singular vectors, corresponding to the forcing and the response operators, at frequency $\tilde{\omega} /(2 \pi)=838.1 \mathrm{~Hz}$, in the plenum (red) and chamber (black). (a) Spatial distribution of the forcing function, (b) phase of the forcing function, (c) spatial distribution of the response function, and (d) phase of the response function.

the system, shown in figure 6 , even though the forcing exhibits a different phase relation between the chamber and plenum modes, when compared to the phase relation of the same eigenmode, suggesting that input disturbances that differ from the systems modal behavior, are capable of exciting a modal response on account of the system's non-normal behavior.

The root-of-unity $j=1$ is associated with degenerate eigenvalues of dimension two. As demonstrated by Ghirardo et al. (2016), these degenerate modes form the building blocks of the resulting limit-cycles, and the manner in which they interact can lead to spinning or standing waves in the combustion chamber. The large non-modal response that is observed for these same modes, and the similarity between the non-modal shapes and eigenmodes, suggests that the least stable low-frequency azimuthal modes (see figure 5b) of this dynamical system can play an important role in the system's longtime behavior. However, the non-normal response is significant only in cases where the nonlinear behavior of the system exhibits a subcritical Hopf bifurcation (Sujith et al. 
2016) as the system parameters are varied across the $(j=1)$-neutral curve, shown in figure 7. The subcritical bifurcation in turn implies the existence of a bi-stable region. This region includes a stable large amplitude limit-cycle accessible even in the state of linear stability, provided that perturbations about the quiescent base-flow elicit a large non-normal response. Therefore, the next section $\S 4.3$ uses the method of multiple scales in order to analyze the weakly nonlinear behavior of the system, with parameters in the vicinity of the $(j=1)$-neutral curve.

\subsection{Weakly nonlinear analysis}

We proceed by describing the conditions which allow broadband, environmental noise to pulse an annular PBC-type combustion chamber, from a stable base-flow to a state of sustained oscillations - a process known as triggering (Sujith et al. 2016) - by analyzing the weakly nonlinear behavior of the system close to the onset of linear instability (Hopf point) using the method of multiple scales. This method determines the nonlinear nature of the Hopf bifurcation and characterizes the branch of periodic solutions that emerge from the Hopf point in the form of a uniformly-valid asymptotic expansion. This is achieved by introducing different time scales to formally separate the fast and slow system behavior as described in Newell \& Whitehead (1969), followed by the superposition of different spatial modes satisfying the system's behavior at each time scale, along with their corresponding time-varying amplitudes. The time-dependent amplitude evolution is described by ordinary differential equations, known as the Stuart-Landau (S-L) equations that result from solvability conditions (Oden \& Demkowicz 2010) for a uniformly valid asymptotic expansion. Finally, the fixed points of these S-L equations determine the amplitude and stability of steady and periodic solutions.

\subsubsection{Asymptotic expansion}

The equation describing the temporal evolution of thermo-acoustic instabilities in an annular combustion chamber, with $N$ identical sectors along the azimuthal direction, can be represented by

$$
\dot{\boldsymbol{x}}(t)=\boldsymbol{A} \boldsymbol{x}(t)+n \sum_{m=1}^{N} \boldsymbol{B}_{m} \sum_{i=1}^{5} \alpha_{i}\left(\boldsymbol{C}_{m} \boldsymbol{x}(t-\tau)\right)^{i},
$$

where, $\boldsymbol{x}$ is the global state vector of the entire $N$-periodic geometry (a union of $\boldsymbol{x}_{u s}$ from each unit-sector), and $\boldsymbol{A}$ is the respective global $N$-periodic block circulant acoustic operator. The term $\alpha_{i}$ represents the coefficients of the nonlinear flame model, as mentioned in equation (2.6), $n$ is the flame strength parameter, and $\tau$ is the flame delay parameter. Column vector $\boldsymbol{B}_{m}$ and row vector $\boldsymbol{C}_{m}$ represent the vectors that select the pressure point at which the compact nonlinear flame model is imposed and the velocity point at which the upstream velocity is measured, respectively.

Once a dynamical system goes through a Hopf bifurcation the existing perturbations are prone to grow and saturate into a limit-cycle due to the existence of higher-order nonlinear terms. If the system exhibits a subcritial bifurcation, then there exists a bistable region ahead of the Hopf point where stable solutions with finite amplitude oscillations appear. The amplitude of these oscillations is extracted using a weakly nonlinear analysis where the governing equation is expanded about the Hopf point, leading to an expansion of the following form

$$
\boldsymbol{x}=\epsilon \boldsymbol{x}_{1}+\epsilon^{2} \boldsymbol{x}_{2}+\epsilon^{3} \boldsymbol{x}_{3}+\ldots
$$

where, $0<\epsilon \ll 1$. The bifurcation parameter is also perturbed around its critical value $n=n^{c}+\Delta n$, where $|\Delta n| \ll 1$. Similar to Orchini et al. (2016), the method 
of multiple scales is then employed, where independent time scales are assumed to be present simultaneously. The fast time scale $t_{0}$ is associated with oscillations of the marginally stable frequency, whereas, time scale $t_{2}$ applies to long-time saturation or growth processes, resulting in

$$
\dot{\boldsymbol{x}}=\frac{\partial \boldsymbol{x}}{\partial t_{0}}+\epsilon^{2} \frac{\partial \boldsymbol{x}}{\partial t_{2}} .
$$

Applying equation (4.3) to governing equations (4.1), we obtain a Taylor expansion of the dynamical system around the fixed point solution $\boldsymbol{x}=0$, at the critical flame strength $n^{c}$ (Hopf point), as follows

$$
\begin{aligned}
\frac{\partial \boldsymbol{x}}{\partial t_{0}}+\epsilon^{2} \frac{\partial \boldsymbol{x}}{\partial t_{2}}=\boldsymbol{A} \boldsymbol{x}(t)+n^{c} \sum_{m=1}^{N} \boldsymbol{B}_{m} \sum_{i=1}^{5} \alpha_{i}\left(\boldsymbol{C}_{m} \boldsymbol{x}(t-\tau)\right)^{i} & \\
& +\Delta n \sum_{m=1}^{N} \boldsymbol{B}_{m} \sum_{i=1}^{5} \alpha_{i}\left(\boldsymbol{C}_{m} \boldsymbol{x}(t-\tau)\right)^{i},
\end{aligned}
$$

where, $\epsilon \approx 10^{-2}$ and $\Delta n=\epsilon^{2} \delta_{2}$ is the $\epsilon^{2}$-order change in the flame strength parameter (Orchini et al. 2016). The term $\delta_{2}$ can take values \pm 1 depending on the direction in which the critical parameter $(n)$ is perturbed. This order of change in the flame strength parameter is chosen so as to obtain control parameters that help enforce solvability conditions (removal of secular forcing terms), which guarantee the validity of the asymptotic expansion at larger time scales. In the case of a subcritical bifurcation, an expansion up to third order resolves oscillations on the unstable branch of the bifurcation diagram; however, these solutions are rarely observed in experiments. In cases where stable limit-cycles prior to the Hopf point are of interest, an expansion up to fifth order is required. Since, the objective of this study is to illustrate the presence of a subcritical bifurcation only, a fifth-order expansion is not considered. In the following sections we solve for each term in the asymptotic expansion mentioned in equation (4.2) one order at a time.

\subsection{2. $\mathcal{O}(\epsilon)$ : eigenvalue problem}

Collecting $\mathcal{O}(\epsilon)$ terms of equation (4.4) results in the linearized version of equation (4.1),

$$
\frac{\partial \boldsymbol{x}_{1}}{\partial t_{0}}-\boldsymbol{A} \boldsymbol{x}_{1}-\sum_{m=1}^{N} \alpha_{1} n^{c} \boldsymbol{B}_{m}\left(\boldsymbol{C}_{m} \boldsymbol{x}_{1}(t-\tau)\right)=0 .
$$

In order to simplify the notation, an operator $\boldsymbol{M}_{s}$ is defined in the spectral domain, and is given by

$$
\boldsymbol{M}_{s}=s \boldsymbol{I}-\boldsymbol{A}-\alpha_{1} n^{c} \sum_{m=1}^{N} \boldsymbol{B}_{m} \otimes \boldsymbol{C}_{m} e^{-s \tau} .
$$

Using $\boldsymbol{M}_{s}$, equation (4.5) can be reformulated as the following nonlinear eigenvalue problem $\boldsymbol{M}_{s} \boldsymbol{x}_{1}=0$. The values of $s$ that satisfy this problem are computed using PDE-method presented in $\S 3$.4. Since each operator $\boldsymbol{I}, \boldsymbol{A}$ and $\boldsymbol{B}_{m} \otimes \boldsymbol{C}_{m}$ is individually block circulant, $\boldsymbol{M}_{s}$ becomes a block circulant operator as well. The analysis presented in Schmid et al. (2017), shows that $\boldsymbol{M}_{s}$ can be transformed into a block diagonal form through a similarity transformation. Thus a block circulant matrix, $\boldsymbol{M}_{s}$, with degenerate eigenvalues, is recast as $N$ root-of-unity matrices (with $N$ unique blocks in the block circulant structure of the direct matrix), each with an eigenspectrum of 
algebraic multiplicity one. Amongst these $N$ operators, there exist $N / 2-1$ or $(N-1) / 2$ pairs of operators that share the same eigenspectrum when $N$ is even or odd, on account of the discrete rotational symmetry associated with the $N$-periodic geometry of the annular combustion chamber (Salas 2013). Since the eigenspectrum of the global block circulant operator is the union of eigenspectra from each linearly independent root-of-unity operator, the global operator must also have degenerate eigenvalues with a maximum algebraic multiplicity of two, in cases where the eigenvalue is non-zero. This implies that the eigenvalue problem in equation (4.5) is simultaneously satisfied by two mutually orthogonal eigenvectors corresponding to two different root-of-unity operators. In other words, any linear combination of the eigenvectors from the root-ofunity operators corresponding to $j$ and $N-j$, that share the same eigenvalue on the verge of a Hopf bifurcation, will also satisfy equation (4.5). For more details regarding the degenerate behavior of such systems, the reader is referred to Salas (2013).

This degeneracy is known to exist for such annular systems, as observed by Ghirardo et al. (2016), where a pair of orthogonal standing modes is used to illustrate the importance of accounting for degenerate eigenmodes in explaining the rich dynamical features, such as spinning, standing and mixed-mode limit-cycles, in a similar annular configuration. While multiple-time-scale analysis has been performed in the past to include nonlinearities in thermo-acoustic systems, such as the work of Orchini et al. (2016), it has mainly focused on longitudinal burner configurations, where degenerate waves are absent. Therefore, the analysis described in Orchini et al. (2016) needs to be modified to include degeneracies in order to be applicable to annular geometries. This modification is achieved by constructing a unique pair of orthogonal eigenvectors $\boldsymbol{x}_{1}^{W}$ and $\boldsymbol{x}_{1}^{Z}$, using the two root-of-unity operators which share the eigenvalue of the global matrix, $\boldsymbol{M}_{s}$, close to a Hopf bifurcation $\left(s=\mathbf{i} \omega^{c}\right)$. The resulting pair of eigenvectors span the space of all vectors satisfying equation (4.5) through the following expression,

$$
\boldsymbol{x}_{1}=\left(W\left(t_{2}\right) \boldsymbol{x}_{1}^{W} e^{\mathrm{i} \omega^{c} t_{0}}+\text { c.c. }\right)+\left(Z\left(t_{2}\right) \boldsymbol{x}_{1}^{Z} e^{\mathbf{i} \omega^{c} t_{0}}+\text { c.c. }\right),
$$

where $W$ and $Z$ are the slowly varying amplitudes of the orthogonal vectors $\boldsymbol{x}_{1}^{W}$ and $\boldsymbol{x}_{1}^{Z}$, respectively. Accounting for a pair of orthogonal eigenvectors rather than a single eigenvector, and the subsequent computation of the higher-order terms incorporates the inherent degeneracy into the final, uniformly valid asymptotic analysis.

\subsection{3. $\mathcal{O}\left(\epsilon^{2}\right)$ : mean shift and second harmonic}

At this order we obtain the equations for the evolution of $\boldsymbol{x}_{2}$. From here onwards, forcing terms appear on the right-hand-side of the equations. These forcing terms are due to interactions between solutions $\boldsymbol{x}_{k}$, where $k<\mathcal{M}$, and $\mathcal{M}$ is the order of the expansion. The only forcing term at this order is $\Sigma_{m=1}^{N} \alpha_{2} n^{c} \boldsymbol{B}_{m}\left(\boldsymbol{C}_{m} \boldsymbol{x}_{1}(t-\tau)\right)^{2}$, which is further expanded, using equation (4.7), to obtain the following $\mathcal{O}\left(\epsilon^{2}\right)$ inhomogeneous 
linear component of equation (4.1)

$$
\begin{aligned}
\frac{\partial \boldsymbol{x}_{2}}{\partial t_{0}}-\boldsymbol{A} \boldsymbol{x}_{2}- & \sum_{m=1}^{N} \alpha_{1} n^{c} \boldsymbol{B}_{m}\left(\boldsymbol{C}_{m} \boldsymbol{x}_{2}(t-\tau)\right) \\
= & \sum_{m=1}^{N} \alpha_{2} n^{c} \boldsymbol{B}_{m}\left(\boldsymbol { C } _ { m } \left(\left(W\left(t_{2}\right) \boldsymbol{x}_{1}^{W} e^{\mathrm{i} \omega^{\mathrm{c}} \mathrm{t}_{0}}+\text { c.c. }\right)\right.\right. \\
& \left.\left.+\left(Z\left(t_{2}\right) \boldsymbol{x}_{1}^{Z} e^{\mathrm{i} \omega^{\mathrm{c}} \mathrm{t}_{0}}+\text { c.c. }\right)\right)\right)^{2} \\
= & |W|^{2}\left(\sum_{m=1}^{N} \boldsymbol{F}_{2, m}^{|W|^{2}}\right)+\left(W^{2}\left(\sum_{m=1}^{N} \boldsymbol{F}_{2, m}^{W^{2}}\right) e^{2 \mathrm{i} \omega^{\mathrm{c}} \mathrm{t}_{0}}+\text { c.c. }\right) \\
& +|Z|^{2}\left(\sum_{m=1}^{N} \boldsymbol{F}_{2, m}^{|Z|^{2}}\right)+\left(Z W^{*}\left(\sum_{m=1}^{N} \boldsymbol{F}_{2, m}^{Z W^{*}}\right)+c . c .\right) \\
& +\left(Z^{2}\left(\sum_{m=1}^{N} \boldsymbol{F}_{2, m}^{Z^{2}}\right) e^{2 \mathrm{i} \omega^{\mathrm{c}} \mathrm{t}_{0}}+\text { c.c. }\right) \\
& +\left(W Z\left(\sum_{m=1}^{N} \boldsymbol{F}_{2, m}^{W Z}\right) e^{2 \mathrm{i} \omega^{\mathrm{c}} \mathrm{t}_{0}}+c . c .\right) .
\end{aligned}
$$

which arises due to the self interaction of $\boldsymbol{x}_{1}$. Explicit expressions for the forcing terms are relegated to the Appendix. The last four terms on the right-hand-side of equation (4.8) are added to the expression derived by Orchini et al. (2016) due to the presence of degeneracy in the annular system. The first two of these terms are modifications to the steady-state forcing, with zero frequency, while the last two represent contribution to the second harmonic. A steady-state solution $\boldsymbol{x}_{2}$, with the same shape as the forcing, can be extracted by using the ansatz,

$$
\begin{aligned}
\boldsymbol{x}_{2}= & |W|^{2}\left(\boldsymbol{x}_{2}^{|W|^{2}}\right)+\left(W^{2}\left(\boldsymbol{x}_{2}^{W^{2}}\right) e^{2 \mathrm{i} \omega^{\mathrm{c}} \mathrm{t}_{0}}+\text { c.c. }\right) \\
& +|Z|^{2}\left(\boldsymbol{x}_{2}^{|Z|^{2}}\right)+\left(Z^{2}\left(\boldsymbol{x}_{2}^{Z^{2}}\right) e^{2 \mathrm{i} \omega^{\mathrm{c}} \mathrm{t}_{0}}+\text { c.c. }\right) \\
& +\left(Z W^{*}\left(\boldsymbol{x}_{2}^{Z W^{*}}\right)+\text { c.c. }\right)+\left(Z W\left(\boldsymbol{x}_{2}^{Z W}\right) e^{2 \mathrm{i} \omega^{c} \mathrm{t}_{0}}+\text { c.c }\right) .
\end{aligned}
$$

Substituting this expression into equation (4.8) and computing each term on the righthand-side in the spectral domain gives,

$$
\left(s \boldsymbol{I}-\boldsymbol{A}-\sum_{m=1}^{N} \alpha_{1} n^{c} \boldsymbol{B}_{m}\left(\boldsymbol{C}_{m} \boldsymbol{x}_{1} e^{-s \tau}\right)\right) \boldsymbol{x}_{2}^{a} e^{s t_{0}}=\boldsymbol{M}_{s} \boldsymbol{x}_{2}^{a} e^{s t_{0}}=\sum_{m=1}^{N} \boldsymbol{F}_{2, m}^{a} e^{s t_{0}},
$$

where $a$ is a general representation of the amplitude-based classification of the terms in the steady-state ansatz, $\boldsymbol{F}_{2, m}^{a}$ represents the amplitude based classification of the righthand-side forcing terms in equation $(4.8 \mathrm{~b})$ and $s$ is the corresponding frequency associated with that term. The matrix $\boldsymbol{M}_{s}$ on the left-hand side is non-singular if $s=2 \mathbf{i} \omega^{c}$ as this is not an eigenvalue of the linear acoustic operator $\boldsymbol{A}$. In case of a constant mode (with zero frequency), the Moore-Penrose inverse is used when evaluating equation (4.10), to ensure that the summation over the force terms is orthogonal to the null space of the adjoint of $\boldsymbol{M}_{0}$. 


\subsection{4. $\mathcal{O}\left(\epsilon^{3}\right)$ : third harmonic and saturation}

At this order the equations for the evolution of $\boldsymbol{x}_{3}$ are obtained. We have

$$
\begin{aligned}
\frac{\partial \boldsymbol{x}_{3}}{\partial t_{0}}-\boldsymbol{A} \boldsymbol{x}_{3}-\sum_{m=1}^{N} \alpha_{1} n^{c} \boldsymbol{B}_{m}\left(\boldsymbol{C}_{m} \boldsymbol{x}_{3}(t-\tau)\right) \\
=-\frac{\partial \boldsymbol{x}_{1}}{\partial t_{2}}-\tau \sum_{m=1}^{N} \alpha_{1} n^{c} \boldsymbol{B}_{m}\left(\boldsymbol{C}_{m} \frac{\partial \boldsymbol{x}_{1}}{\partial t_{2}}(t-\tau)\right) \\
\quad+\left(\boldsymbol{F}_{\omega^{c}}\left(\boldsymbol{x}_{1}, \boldsymbol{x}_{2}\right) e^{\mathrm{i} \omega^{c} \mathrm{t}_{0}}+\boldsymbol{F}_{3 \omega^{c}}\left(\boldsymbol{x}_{1}, \boldsymbol{x}_{2}\right) e^{3 \mathrm{i} \omega^{c} \mathrm{t}_{0}}+\text { c.c. }\right) .
\end{aligned}
$$

Following the procedure from the previous section, expressions in equations (4.7) and (4.9) are used to obtain an amplitude-based classification of the forcing terms as demonstrated in Appendix. Explicit expressions for the forcing terms are also relegated to the Appendix.

Forcing terms corresponding to the angular frequency $\omega^{c}$ (equation B 2) lead to resonant forcing at eight different amplitude levels, namely $W,|W|^{2} W, Z,|Z|^{2} Z,|W|^{2} Z$, $|Z|^{2} W, W^{2} Z^{*}$ and $Z^{2} W^{*}$. Hence, a solvability condition - also known as the Fredholm alternative (Oden \& Demkowicz (2017)) - needs to be satisfied in order for a uniformly valid, asymptotic solution to exist. This solvability condition requires the sum of the resonant forcing terms to be orthogonal to the kernel of the adjoint matrix $\boldsymbol{M}_{\mathbf{i} \omega^{c}}^{+}$(Sipp \& Lebedev 2007). This procedure generalizes the idea of canceling the secular terms, employed for weakly nonlinear analysis of scalar problems (Orchini et al. 2016).

In order to derive this solvability condition, a set of unique vectors spanning the null space of the Hermitian of the direct matrix $\boldsymbol{M}_{\mathbf{i} \omega^{c}}$, or in other words its adjoint, are required. The direct matrix under consideration is known to have eigenvalues that have an algebraic multiplicity of two and is block circulant, thus ensuring its adjoint (Hermitian) shares these properties. The equivalent procedure that was used to determine $\boldsymbol{x}_{1}^{W}$ and $\boldsymbol{x}_{1}^{Z}$ is employed to construct a pair of orthogonal eigenvectors, denoted by $\boldsymbol{x}_{1}^{W+}$ and $\boldsymbol{x}_{1}^{Z+}$, associated with the nullspace of the adjoint matrix, by simply considering the eigenvectors of the Hermitian of the two root-of-unity matrices, used to obtain $\boldsymbol{x}_{1}^{W}$ and $\boldsymbol{x}_{1}^{Z}$. Applying this solvability condition, described in detail in the appendix, leads to the following coupled equations describing the evolution of the complex amplitudes $W$ and $Z$ :

$$
\begin{aligned}
\beta_{\partial W} \frac{\partial W}{\partial t_{2}}+\beta_{\partial Z} \frac{\partial Z}{\partial t_{2}}= & \beta_{W} W+\beta_{|W|^{2} W}|W|^{2} W+\beta_{Z} Z+\beta_{|Z|^{2} Z}|Z|^{2} Z+\beta_{|W|^{2} Z}|W|^{2} Z \\
& +\beta_{|Z|^{2} W}|Z|^{2} W+\beta_{W^{2} Z^{*}} W^{2} Z^{*}+\beta_{Z^{2} W^{*}} Z^{2} W^{*} \\
\gamma_{\partial W} \frac{\partial W}{\partial t_{2}}+\gamma_{\partial Z} \frac{\partial Z}{\partial t_{2}}= & \gamma_{W} W+\gamma_{|W|^{2} W}|W|^{2} W+\gamma_{Z} Z+\gamma_{|Z|^{2} Z}|Z|^{2} Z+\gamma_{|W|^{2} Z}|W|^{2} Z \\
& +\gamma_{|Z|^{2} W}|Z|^{2} W+\gamma_{W^{2} Z^{*}} W^{2} Z^{*}+\gamma_{Z^{2} W^{*}} Z^{2} W^{*}
\end{aligned}
$$

The coefficients $\beta_{a}$ (or $\gamma_{a}$ ) are given as the inner products between $\boldsymbol{x}_{1}^{W+}$ (or $\boldsymbol{x}_{1}^{Z+}$ ) and $\sum_{m=1}^{N} \boldsymbol{F}_{3, m}^{a}$ where $a$ is a general representation of amplitude.

\subsubsection{Stuart-Landau equation at $\mathcal{O}\left(\epsilon^{3}\right)$ and flame model}

Equations (4.12) are known as Stuart-Landau equations. Together, these equations yield the amplitude of limit-cycle solutions and the frequency shift (not mentioned here) 
of the nonlinear oscillation with respect to the marginally stable eigenfrequency. In other words, these equations help construct the nonlinear mechanism which describes the finiteamplitude thermo-acoustic oscillations in annular combustors by providing ODE's that describe the temporal variation of the complex amplitudes $W\left(t_{2}\right)$ and $Z\left(t_{2}\right)$.

Using a polar representation $W=r_{W} e^{\mathbf{i} \theta_{W}}, Z=r_{Z} e^{\mathbf{i} \theta_{Z}}$ and observing that the terms $\beta_{\partial Z}, \gamma_{\partial W}, \beta_{Z}$ and $\gamma_{W}$ are zero on account of the different root-of-unity indices used to construct the global versions of these vectors gives

$$
\begin{aligned}
\frac{\partial r_{W}}{\partial t_{2}}= & \Re\left(\frac{\beta_{W}}{\beta_{\partial W}}\right) r_{W}+\Re\left(\frac{\beta_{|W|^{2} W}}{\beta_{\partial W}}\right) r_{W}^{3}+\Re\left(\frac{\beta_{|Z|^{2} Z}}{\beta_{\partial W}}\right) r_{Z}^{3}+\Re\left(\frac{\beta_{|W|^{2} Z}}{\beta_{\partial W}}\right) r_{W}^{2} r_{Z} \\
& +\Re\left(\frac{\beta_{|Z|^{2} W}}{\beta_{\partial W}}\right) r_{Z}^{2} r_{W}+\Re\left(\frac{\beta_{W^{2} Z^{*}}}{\beta_{\partial W}}\right) r_{W}^{2} r_{Z}+\Re\left(\frac{\beta_{Z^{2} W^{*}}}{\beta_{\partial W}}\right) r_{Z}^{2} r_{W}, \\
\frac{\partial r_{Z}}{\partial t_{2}}= & \Re\left(\frac{\gamma_{Z}}{\gamma_{\partial Z}}\right) r_{Z}+\Re\left(\frac{\gamma_{|W|^{2} W}}{\gamma_{\partial Z}}\right) r_{W}^{3}+\Re\left(\frac{\gamma_{|Z|^{2} Z}}{\gamma_{\partial Z}}\right) r_{Z}^{3}+\Re\left(\frac{\gamma_{|W|^{2} Z}}{\gamma_{\partial Z}}\right) r_{W}^{2} r_{Z} \\
& +\Re\left(\frac{\gamma_{|Z|^{2} W}}{\gamma_{\partial Z}}\right) r_{Z}^{2} r_{W}+\Re\left(\frac{\gamma_{W^{2} Z^{*}}}{\gamma_{\partial Z}}\right) r_{W}^{2} r_{Z}+\Re\left(\frac{\gamma_{Z^{2} W^{*}}}{\gamma_{\partial Z}}\right) r_{Z}^{2} r_{W} .
\end{aligned}
$$

These equations are then used to find the steady-state and periodic solutions by computing the amplitudes $r_{W}$ and $r_{Z}$ in the asymptotic limit $t_{2} \rightarrow \infty$; in other words the roots of the above equations are the steady-state or periodic solutions of the underlying dynamical system. Amplitudes found with $\operatorname{sign}(\Delta n)=\delta_{2}=-1$ signify a subcritical bifurcation and those found with $\operatorname{sign}(\Delta n)=\delta_{2}=1$ describe a supercritical bifurcation. In the case of a subcritical (supercritical) bifurcation, the extracted amplitude correspond to an unstable (stable) limit-cycle, when the weakly nonlinear asymptotic expansion is truncated at $\mathcal{O}\left(\epsilon^{3}\right)$. As mentioned previously, to compute the amplitude of a stable limitcycle in case of a subcritical bifurcation, the weakly nonlinear expansion must include fourth- and fifth-order terms.

At this stage, the neutral curve displayed in figure 7 can further be analyzed to determine the nature of the bifurcation by studying the roots of its representative StuartLandau equation. However, in order to perform the weakly nonlinear expansion, a suitable nonlinear flame model needs to be selected. This flame model is derived using the same least-squares procedure described in Orchini et al. (2016), approximating the saturationtype nonlinearity displayed by King's law, as illustrated in figure 11. The flame model coefficients used to generate this comparison were given in equation set 2.7. This model is similar to the flame model used in (Orchini et al. 2016), with a slightly different domain. In our study, the domain $[-1.839,1.839]$ is chosen. This domain is sufficiently wide to include the saturation mechanism for amplitudes much higher than those that will be considered in this study. Figure 11(a) shows that the least-square fit model has the same linear behavior as the unsteady King's law and a qualitatively similar nonlinear behavior, with a smooth saturation mechanism. When displaying the gain $G$, shown in figure 11(b), the gain monotonically decreases with the amplitude forcing, except for a small region of $u / \tilde{u}<0.2$, where the value slightly increases. The modest gain at small velocity amplitudes promotes the occurrence of a subcritical bifurcation over the portion of the neutral curve, corresponding to root-of-unity index $j=1$, displayed in figure 14 .

\subsubsection{Weakly nonlinear analysis of the Hopf bifurcation}

We then apply the weakly nonlinear analysis to the annular PBC configuration, described in $\S 2$, in order to ascertain the nature of the systems Hopf bifurcation and hence the evolution of finite-amplitude thermo-acoustic instabilities. At first, we focus on the weakly nonlinear behavior at a single point adjacent to the neutral curve, with 


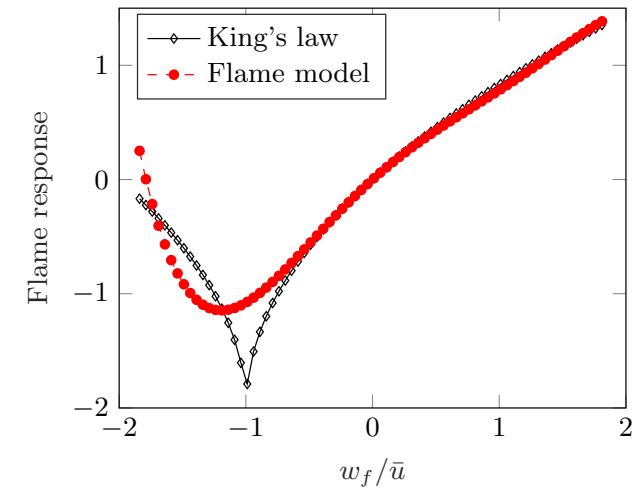

(a)

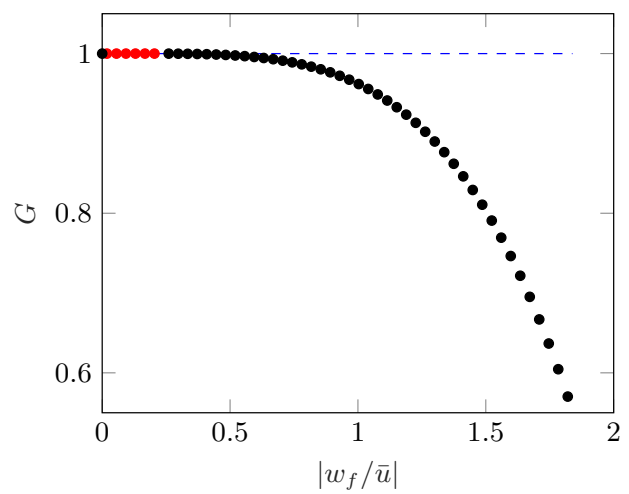

(b)

Figure 11. (a) Comparison of flame response from the least-squares flame model (red) to that of King's law (black). (b) Gain of the flame model as a function of the velocity perturbation magnitudes, (red) signifies the portion with gain larger than unity. Here, $w_{f}$ is the velocity perturbation upstream (cold side) of the flame.

parameter values of $n=0.2425-\epsilon^{2}$, and $\tau=3.85$, where $\epsilon=10^{-3}$. As mentioned in section 4.2 , in $(n, \tau)$ space this point lies a distance of $\epsilon^{2}$ to the left of the black square in figure 7 and corresponds to the location where nonmodal instabilities of the PBC system have been analyzed. Figure 12 shows a phase portrait of the temporally evolving finite-amplitude perturbations, along with the roots of the underlying Stuart-Landau equation for the selected configuration. The point at the origin represents the steadystate solution of the system, illustrated by the converging streamlines in its vicinity, whereas the other two points indicate a pair of spinning modes. Figure 12 shows that, close to these periodic solutions, finite-amplitude perturbations are both attracted and repelled. This is characteristic of a saddle-type finite-amplitude perturbation or a limit cycle, revealing the bi-stable nature of the system's behavior close to its neutral curve. The amplitude of the resulting unstable limit cycle, reported in the form of the axial velocity perturbations upstream of the flame, can now be extracted using the fixed point values of $r_{W}$ and $r_{Z}$ as

$$
\left|\boldsymbol{C}_{1} \cdot \boldsymbol{x}\right|=\left|\boldsymbol{C}_{1} \cdot\left(\epsilon \boldsymbol{x}_{1}(W, Z)+\epsilon^{2} \boldsymbol{x}_{2}(W, Z)+\epsilon^{3} \boldsymbol{x}_{3}(W, Z)\right)\right|
$$

where, $W=r_{W} e^{i \theta_{W}}, Z=r_{Z} e^{i \theta_{Z}}$ and, $\boldsymbol{x}_{1}(W, Z), \boldsymbol{x}_{2}(W, Z)$ and $\boldsymbol{x}_{3}(W, Z)$ are given by expressions (4.7), (4.9) and (B 3), respectively. Therefore, for the choice of parameters $n=0.2425$ and $\tau=3.85$, weakly nonlinear analysis suggests the presence of a subcritical bifurcation, and hence, the existence of an unstable limit cycle with oscillation amplitude $\left|\boldsymbol{C}_{1} \cdot \boldsymbol{x}\right|=0.0473$.

In order to verify the result of the weakly nonlinear analysis, the governing equations, given in (4.1), are linearized about their limit cycle solution, taken from the weakly nonlinear analysis, where $\boldsymbol{x}_{L C}=\epsilon \boldsymbol{x}_{1}+\epsilon^{2} \boldsymbol{x}_{2}+\epsilon^{3} \boldsymbol{x}_{3}$,

$$
\begin{aligned}
\boldsymbol{L} \boldsymbol{x}^{1}=\boldsymbol{A} \boldsymbol{x}^{1} & +\sum_{m=1}^{N}\left(( n ^ { c } - \epsilon ^ { 2 } ) \left(\alpha_{1}+2 \alpha_{2}\left|\boldsymbol{C}_{m} \cdot \boldsymbol{x}_{L C}\right| \cos \left(\omega^{c}(t-\tau)\right)\right.\right. \\
& \left.\left.+3 \alpha_{3}\left|\boldsymbol{C}_{m} \cdot \boldsymbol{x}_{L C}\right|^{2} \cos ^{2}\left(\omega^{c}(t-\tau)\right)\right) \boldsymbol{B}_{m} \boldsymbol{C}_{m}^{T}\right) \boldsymbol{x}^{1}(t-\tau) .
\end{aligned}
$$

This is a non-autonomous differential equation, where $\boldsymbol{x}^{1}$ denotes small-amplitude perturbations about the limit cycle solution $\boldsymbol{x}_{L C}$. The stability of such an equation is 


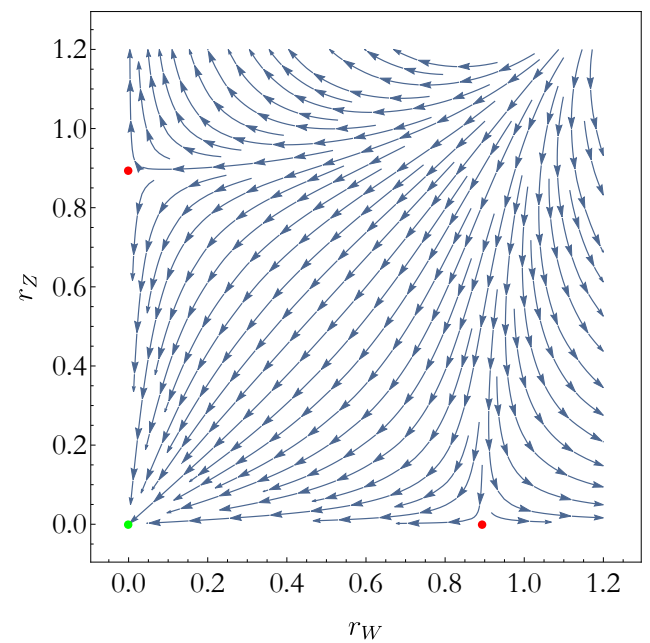

FIGURE 12. Phase portrait of the time evolution of finite amplitude perturbations within the PBC configuration. Parameters $n=0.2425-\epsilon^{2}, \tau=3.85$ and $\epsilon=10^{-2}$.

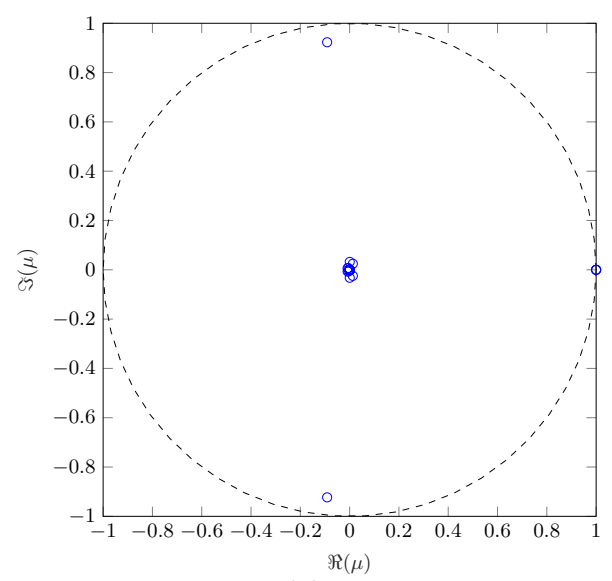

(a)

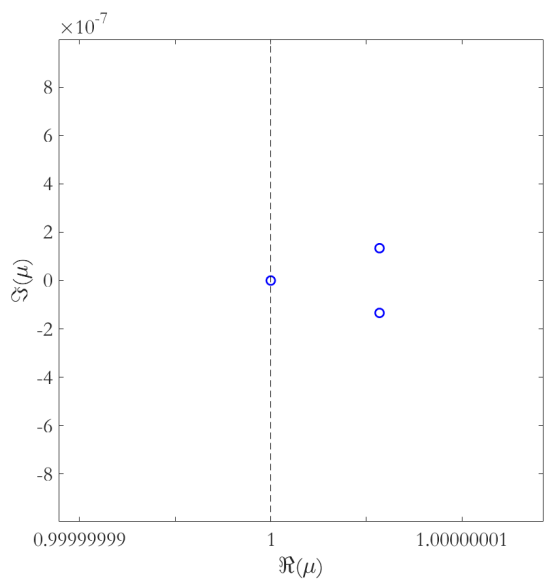

(b)

Figure 13. (a) Flouqet multipliers of small amplitude perturbations to the equation (4.15) (b) Enlarged view of the Floquet multipliers close to the unity gain point at $1+0 i$.

characterized by its respective Floquet multipliers. In the case of the PBC configuration, producing the phase diagram of figure 12, three distinct Floquet multipliers $(\mu)$ are present: unstable $(\mu>1)$, neutral $(\mu=1)$ and stable $(\mu<1)$, shown in figure 13 . As demonstrated by Juniper (2011), this confirms the presence of an unstable limit cycle, which repels states in the direction of the eigenvector corresponding to the Floquet multiplier with $\mu>1$, remains neutral in the direction of the periodic solution, and attracts states from every other direction. The Floquet analysis, therefore, verifies the validity of the weakly nonlinear expansion.

At this stage, the subcritical/supercritical nature of the Hopf bifurcation along the neutral curve of figure 7 can be investigated. For this purpose, only the portion of the neutral curve corresponding to a transition in linear stability of the $j=1$ modes is considered, on account of the eigenmodes' inherent degeneracy, as mentioned in $\S 4.2$. 


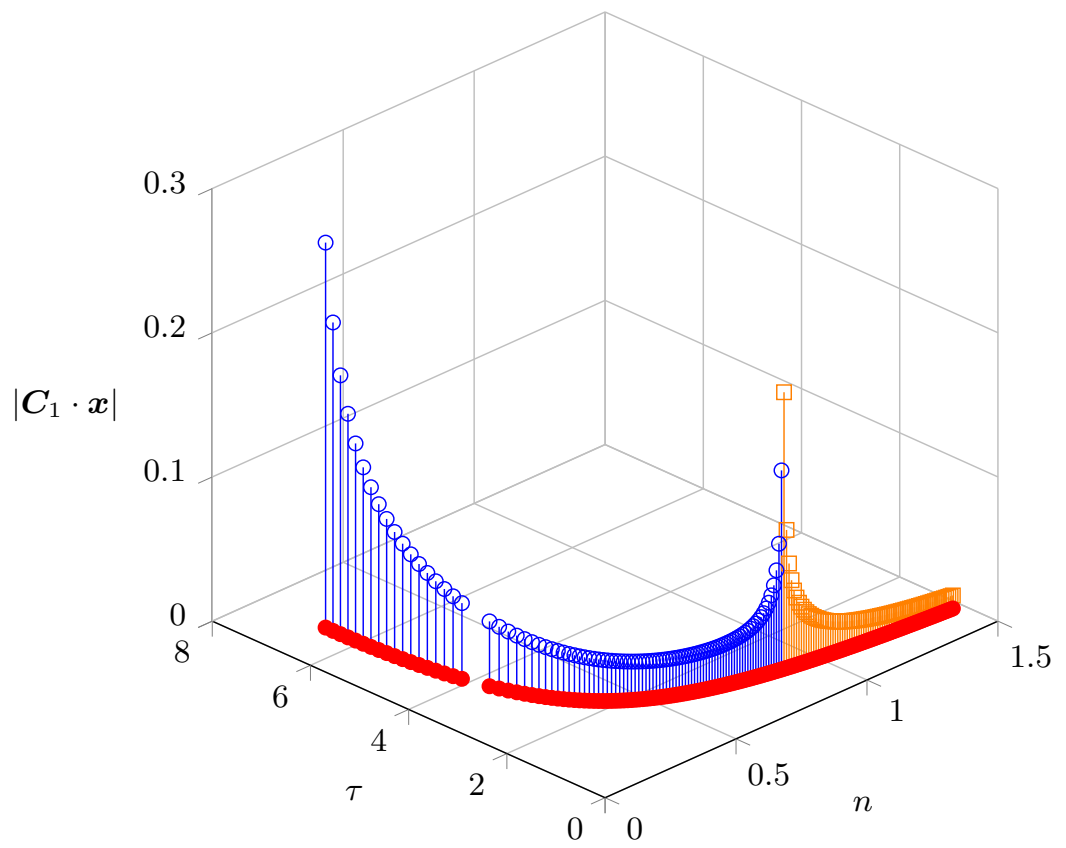

FiguRE 14. Unstable (blue circles) and stable (orange squares) limit cycle amplitude, along the neutral curve corresponding to the roots of unity $j=1$ or $j=N-1$.

The phase difference between the pair of orthogonal spinning modes $\boldsymbol{x}_{1}^{W}$ and $\boldsymbol{x}_{1}^{Z}$ that span the subspace of eigenmodes corresponding to the degenerate eigenvalues during the Hopf bifurcation, is known to influence the phase portrait in figure 12 (Ghirardo et al. 2016). Consequently, the results obtained in this section are extracted with modes which are phase-aligned to yield a pure standing wave when superimposed with the same amplitude.

Figure 14 shows that depending on the values of $\tau$ and $n$ the system can undergo both subcritical and supercritical bifurcations along a neutral curve. As the value of $\tau$ decreases, with increasing values of $n$, the amplitude of the unstable limit cycle in the case of a subcritical bifurcation decreases as it reaches a critical value $(n=0.5558$, $\tau=1.65)$, and then slightly increases. Finally, at the point $(n=0.8895, \tau=1.1)$ the saddle point of the subcritical bifurcation approaches the Hopf bifurcation point, causing a change in the nature of the bifurcation from subcritical to supercritical, which appears as a sudden rise in the amplitude value. Since triggering is only effective in the presence of a subcritical bifurcation, non-normal analysis is relevant for the portion of the neutral curve highlighted in blue in figure 14 .

\section{Summary and conclusions}

The linear and weakly nonlinear stability of an energy conversion device, consisting of circular arrangements of $N$ individual burner units connected by an annular chamber and plenum, is studied using a framework based on the state-space representation of a network-type model. Configurations of this type approximate the internal thermoacoustic dynamics of combustion chambers. The azimuthally periodic nature of an $N$ unit device yields a discrete rotational symmetry which, in turn, translates into a blockcirculant system matrix after the governing equations are discretized. This structure can 
be exploited by a root-of-unit approach (Schmid et al. 2017) which allows the reduction of the full global system to a finite set of single-unit analyses. Modal, non-modal and weakly nonlinear calculations can be conveniently formulated and executed within this framework.

A modal analysis of the resulting system of delay-differential equations shows instabilities that arise in an annular combustor model. Here, the most unstable dynamics consists of multiple individual units locking into a large-scale azimuthal motion. The annular combustor, modeled after the case study described in Laera et al. (2017), shows a clear preference for the root-of-unity index $j=1$ (over a certain region of $n-\tau$ space), before a fully periodic (over one individual unit) motion becomes unstable (see figure 7).

While the combustor configuration can support thermo-acoustic instabilities for specific parameter combinations, it is equally important to assess the amplification potential due to external forcing when operating in an asymptotically stable parameter regime. Mathematically, this has been accomplished by a resolvent analysis - again taking advantage of the block-circulant nature of the governing equations. For parameters that correspond to the square point on figure 7 , the response behavior follows both a resonance argument: (i) frequencies are preferentially amplified, if they nearly coincide with one of the inherent eigenfrequencies of the underlying linear system, and (ii) a strong frequency response may appear at frequencies that are far from instrinsic eigenfrequencies. This latter response behavior has to be attributed to non-modal effects, more specifically to pseudo-resonances; in this case, a substantial response of the linear system can be solicited by a harmonic forcing whose frequency is appreciably far from the eigenfrequencies of the underlying system. Frequency ranges where non-modal response behavior are observed, have been identified for the annular PBC combustion chamber.

To quantify the relevance of the system's non-modal response, a weakly nonlinear analysis demonstrates that the system exhibits a subcritical bifurcation (blue; figure 14) along the $j=1$ neutral curve, hence proving the bistable nature of the steady solution close to a Hopf bifurcation. For this reason, perturbations about the steady base flow along the subcritical part of the neutral curve could transition into a stable limit-cycle solution - even under linearly stable, steady base-flow conditions.

The presented study of combustion systems that consist of $N$ identical subunits (arranged in a circular manner and linked by an exchange of acoustic information) has demonstrated that instabilities - of modal or non-modal nature - can arise that favor a nearest-neighbor synchronization across multiple individual units. The employed mathematical and computational framework is particularly suited to fully and efficiently capture the global dynamics of the studied configurations. More complex fluid, acoustic and thermo-acoustic models that fall within the same geometric classification and are prone to corresponding instabilities and synchronizations, can be analyzed using the same methodology; they will be the focus of future investigations.

\section{Appendix A. Mass conservation at burner-chamber (plenum) junction}

The non-dimensional time derivative of pressure from equation set (2.3) can yield the time derivative of density, in dimensional form, as follows:

$$
\frac{\partial \tilde{\rho}}{\partial \tilde{t}}=-\frac{\gamma p_{0} u_{0, d}}{L_{0} c_{0}^{2}} \nabla \cdot u+\frac{\nu \gamma p_{0} u_{0, d}}{L_{0} c_{0}^{2}} \nabla^{2} p,
$$




\section{Control volume}

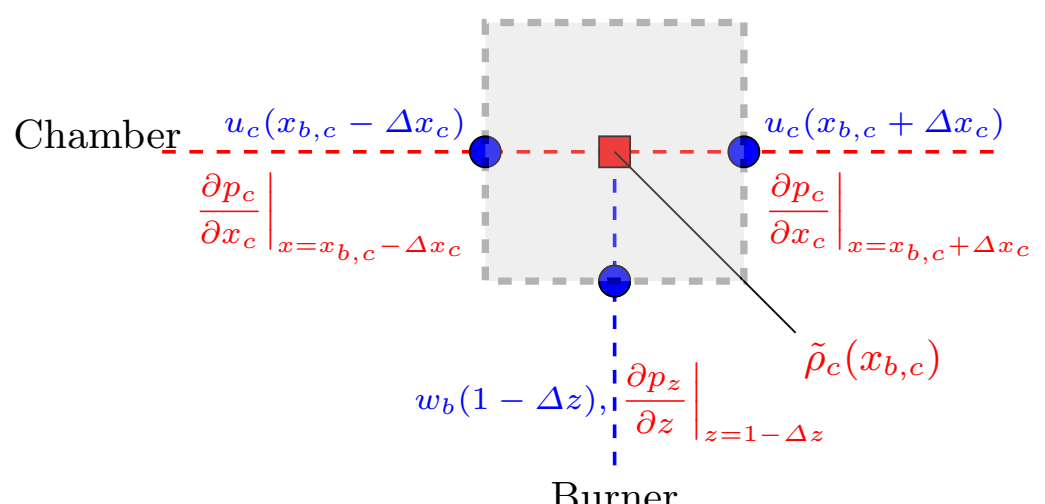

FIGURE 15. The control volume surrounding a chamber-burner junction.

where $c_{0}$ is the local speed of sound at the burner-chamber(plenum) junction $\left(c_{0}=c_{0, u}\right.$ at $z=0$ and $c_{0}=c_{0, d}$ at $z=1$ ). Now, integrating this expression over a control volume $(\mathrm{CV}$; as shown in figure 15) surrounding the junction, time rate of change of mass within the $\mathrm{CV}$ is given by:

$$
\int \frac{\partial \tilde{\rho}}{\partial \tilde{t}} d V=-\frac{\gamma p_{0} u_{0, d}}{L_{0} c_{0}^{2}} \int \nabla \cdot u d V+\frac{\nu \gamma p_{0} u_{0, d}}{L_{0} c_{0}^{2}} \oint \overrightarrow{\nabla p} \cdot \hat{\boldsymbol{n}} d S=0,
$$

where, $\hat{\boldsymbol{n}}$ is the standard outward normal unit vector on the surface of the CV and, $V$ and $S$ have their usual meaning of the volume and surface of the CV, respectively.

Therefore, the time rate of change of density at the junction where the $1 \mathrm{D}$ representations of the burner and chamber (or plenum) meet, is given by:

Burner-chamber junction:

$$
\begin{aligned}
\frac{\partial \tilde{\rho}}{\partial \tilde{t}}= & -\frac{\gamma p_{0} u_{0, d}}{L_{0} c^{2}}\left(\frac{\partial u}{\partial x_{c}}-\frac{w S_{b}}{\int S_{c} d x_{c}}\right) \\
& +\frac{\nu \gamma p_{0} u_{0, d}}{L_{0} c^{2}}\left(\frac{\partial^{2} p}{\partial x_{c}^{2}}-\frac{\left.S_{i}\left(\partial p_{z} / \partial z\right)\right|_{z=1-\Delta z}}{\int S_{c} d x_{c}}\right) \text { when } x_{c}=x_{b, c}
\end{aligned}
$$

Burner-plenum junction:

$$
\begin{aligned}
\frac{\partial \tilde{\rho}}{\partial \tilde{t}}= & -\frac{\gamma p_{0} u_{0, d}}{L_{0} c^{2}}\left(\frac{\partial u}{\partial x_{p}}+\frac{w S_{b}}{\int S_{p} d x_{p}}\right) \\
& +\frac{\nu \gamma p_{0} u_{0, d}}{L_{0} c^{2}}\left(\frac{\partial^{2} p}{\partial x_{p}^{2}}+\frac{\left.S_{i}\left(\partial p_{z} / \partial z\right)\right|_{z=\Delta z}}{\int S_{p} d x_{p}}\right) \text { when } x_{p}=x_{b, p},
\end{aligned}
$$

here, $S_{c}, S_{p}$ and $S_{i}$ are the chamber, plenum and burner cross sectional areas, $x_{c}$ and $x_{p}$ represent the one-dimensional direction along the perimeter in case of the chamber and plenum, $z$ is the longitudinal direction along the burner and, $x_{b, c}$ and $x_{b, p}$ represent the location of the junctions along the chamber and plenum perimeter, respectively. Furthermore, equations (A 3) and (A 4) can be multiplied by $L_{0} c_{0}^{2} /\left(\gamma p_{0} u_{0, d}\right)$ to obtain the time derivative of pressure at the two junctions, as presented in equation set (2.9). 


\title{
Appendix B. Inhomogeneous linear ODE's and corresponding forcing terms
}

\author{
B.1. $\mathcal{O}\left(\epsilon^{2}\right)$ :
}

The second-order forcing terms in the weakly nonlinear expansion are given as

$$
\begin{aligned}
& \boldsymbol{F}_{2, m}^{|W|^{2}}=2 \alpha_{2} K^{c} \boldsymbol{B}_{m}\left|\boldsymbol{C}_{m} \boldsymbol{x}_{1}^{W}\right|^{2}, \\
& \boldsymbol{F}_{2, m}^{|Z|^{2}}=2 \alpha_{2} K^{c} \boldsymbol{B}_{m}\left|\boldsymbol{C}_{m} \boldsymbol{x}_{1}^{Z}\right|^{2}, \\
& \boldsymbol{F}_{2, m}^{W^{2}}=\alpha_{2} K^{c} \boldsymbol{B}_{m}\left(\boldsymbol{C}_{m} \boldsymbol{x}_{1}^{W}\right)^{2} e^{-2 \mathrm{i} \omega^{\mathrm{c}} \tau}, \\
& \boldsymbol{F}_{2, m}^{Z^{2}}=\alpha_{2} K^{c} \boldsymbol{B}_{m}\left(\boldsymbol{C}_{m} \boldsymbol{x}_{1}^{Z}\right)^{2} e^{-2 \mathrm{i} \omega^{\mathrm{c}} \tau}, \\
& \boldsymbol{F}_{2, m}^{Z W^{*}}=2 \alpha_{2} K^{c} \boldsymbol{B}_{m}\left(\boldsymbol{C}_{m} \boldsymbol{x}_{1}^{Z}\right)\left(\boldsymbol{C}_{m} \boldsymbol{x}_{1}^{W}\right)^{*}, \\
& \boldsymbol{F}_{2, m}^{W Z}=2 \alpha_{2} K^{c} \boldsymbol{B}_{m}\left(\boldsymbol{C}_{m} \boldsymbol{x}_{1}^{Z}\right)\left(\boldsymbol{C}_{m} \boldsymbol{x}_{1}^{W}\right) e^{-2 \mathrm{i} \omega^{\mathrm{c}} \tau} . \\
& \text { B. } 2 . \mathcal{O}\left(\epsilon^{3}\right):
\end{aligned}
$$

The $\mathcal{O}\left(\epsilon^{3}\right)$ inhomogeneous linear equation is given by

$$
\begin{aligned}
\frac{\partial \boldsymbol{x}_{3}}{\partial t_{0}} & -\boldsymbol{A}_{3}-\sum_{m=1}^{N} \alpha_{1} n^{c} \boldsymbol{B}_{m}\left(\boldsymbol{C}_{m} \boldsymbol{x}_{3}(t-\tau)\right) \\
& =-\frac{\partial W}{\partial t_{2}}\left(\boldsymbol{x}_{1}^{W} e^{\mathrm{i} \omega^{\mathrm{c}} \mathrm{t}_{0}}+\tau \alpha_{1} n^{c} \sum_{m=1}^{N} \boldsymbol{B}_{m}\left(\boldsymbol{C}_{m} \boldsymbol{x}_{1}^{W} e^{\mathrm{i} \omega^{\mathrm{c}}\left(\mathrm{t}_{0}-\tau\right)}\right)\right)+W\left(\sum_{m=1}^{N} \boldsymbol{F}_{3, m}^{W}\right) e^{\mathrm{i} \omega^{\mathrm{c}} \mathrm{t}_{0}} \\
& +|W|^{2} W\left(\sum_{m=1}^{N} \boldsymbol{F}_{3, m}^{|W|^{2} W}\right) e^{\mathrm{i} \omega^{\mathrm{c}} \mathrm{t}_{0}}+W^{3}\left(\sum_{m=1}^{N} \boldsymbol{F}_{3, m}^{W^{3}}\right) e^{3 \mathrm{i} \omega^{\mathrm{c}} \mathrm{t}_{0}} \\
& -\frac{\partial Z}{\partial t_{2}}\left(\boldsymbol{x}_{1}^{Z} e^{\mathrm{i} \omega^{\mathrm{c}} \mathrm{t}_{0}}+\tau \alpha_{1} n^{c} \sum_{m=1}^{N} \boldsymbol{B}_{m}\left(\boldsymbol{C}_{m} \boldsymbol{x}_{1}^{Z} e^{\mathrm{i} \omega^{\mathrm{c}}\left(\mathrm{t}_{0}-\tau\right)}\right)\right) \\
& +Z\left(\sum_{m=1}^{N} \boldsymbol{F}_{3, m}^{Z}\right) e^{\mathrm{i} \omega^{\mathrm{c}} \mathrm{t}_{0}}+|Z|^{2} Z\left(\sum_{m=1}^{N} \boldsymbol{F}_{3, m}^{|Z|^{2} Z}\right)^{\mathrm{i} \omega^{\mathrm{c}} \mathrm{t}_{0}}+Z^{3}\left(\sum_{m=1}^{N} \boldsymbol{F}_{3, m}^{Z^{3}}\right) e^{3 \mathrm{i} \omega^{\mathrm{c}} \mathrm{t}_{0}} \\
& +|W|^{2} Z\left(\sum_{m=1}^{N} \boldsymbol{F}_{3, m}^{|W|^{2} Z}\right) e^{\mathrm{i} \omega^{\mathrm{c}} \mathrm{t}_{0}}+|Z|^{2} W\left(\sum_{m=1}^{N} \boldsymbol{F}_{3, m}^{|Z|^{2} W}\right) e^{\mathrm{i} \omega^{\mathrm{c}} \mathrm{t}_{0}} \\
& +W W^{2} Z^{*}\left(\sum_{m=1}^{N} \boldsymbol{F}_{3, m}^{W^{2} Z^{*}}\right) e^{\mathrm{i} \omega^{\mathrm{c}} \mathrm{t}_{0}}+Z^{2} W^{*}\left(\sum_{m=1}^{N} \boldsymbol{F}_{3, m}^{Z^{2} W^{*}}\right) e^{\mathrm{i} \omega^{\mathrm{c}} \mathrm{t}_{0}} \\
& +W W^{2} Z\left(\sum_{m=1}^{N} \boldsymbol{F}_{3, m}^{W^{2} Z}\right) e^{3 \mathrm{i} \omega^{\mathrm{c}} \mathrm{t}_{0}}+Z^{2} W\left(\sum_{m=1}^{N} \boldsymbol{F}_{3, m}^{Z^{2} W} e^{3 \mathrm{i} \omega^{\mathrm{c}} \mathrm{t}_{0}}+c . c .\right.
\end{aligned}
$$

Assuming a steady-state solution of the same form as the forcing, we obtain

$$
\begin{aligned}
\boldsymbol{x}_{3}= & W \boldsymbol{x}_{3}^{W} e^{\mathrm{i} \omega^{\mathrm{c}} \mathrm{t}_{0}}+|W|^{2} W \boldsymbol{x}_{3}^{|W|^{2} W} e^{\mathrm{i} \omega^{\mathrm{c}} \mathrm{t}_{0}}+W^{3} \boldsymbol{x}_{3}^{W^{3}} e^{3 \mathrm{i} \omega^{\mathrm{c}} \mathrm{t}_{0}}+Z \boldsymbol{x}_{3}^{Z} e^{\mathrm{i} \omega^{\mathrm{c}} \mathrm{t}_{0}} \\
& +|Z|^{2} Z \boldsymbol{x}_{3}^{|Z|^{2} Z} e^{\mathrm{i} \omega^{\mathrm{c}} \mathrm{t}_{0}}+Z^{3} \boldsymbol{x}_{3}^{Z^{3}} e^{3 \mathrm{i} \omega^{\mathrm{c}} \mathrm{t}_{0}}+|W|^{2} Z \boldsymbol{x}_{3}^{|W|^{2} Z} e^{\mathrm{i} \omega^{\mathrm{c}} \mathrm{t}_{0}}+|Z|^{2} W \boldsymbol{x}_{3}^{|Z|^{2} W} e^{\mathrm{i} \omega^{\mathrm{c}} \mathrm{t}_{0}} \\
& +W^{2} Z^{*} \boldsymbol{x}_{3}^{W^{2} Z^{*}} e^{\mathrm{i} \omega^{\mathrm{c}} \mathrm{t}_{0}}+Z^{2} W^{*} \boldsymbol{x}_{3}^{Z^{2} W^{*}} e^{\mathrm{i} \omega^{\mathrm{c}} \mathrm{t}_{0}}+W^{2} Z \boldsymbol{x}_{3}^{W^{2} Z} e^{3 \mathrm{i} \omega^{\mathrm{c}} \mathrm{t}_{0}} \\
& +Z^{2} W \boldsymbol{x}_{3}^{Z^{2} W} e^{3 \mathrm{i} \omega^{\mathrm{c}} \mathrm{t}_{0}}+\text { c.c. },
\end{aligned}
$$

where each forcing term is explicitly defined in the following equations. For brevity sake 
only half of them are presented below, the remaining terms can be generated by replacing $W$ with $Z$ and visa versa in each term. We have

$$
\begin{aligned}
& \boldsymbol{F}_{3, m}^{W}=\alpha_{1} \delta_{2} \boldsymbol{B}_{m}\left(\boldsymbol{C}_{m} \boldsymbol{x}_{1}^{W}\right) e^{-\mathrm{i} \omega^{\mathrm{c}} \tau}, \\
& \boldsymbol{F}_{3, m}^{|W|^{2} W}=\boldsymbol{B}_{m} n^{c}\left[3 \alpha_{3}\left(\boldsymbol{C}_{m} \boldsymbol{x}_{1}^{W}\right)\left|\left(\boldsymbol{C}_{m} \boldsymbol{x}_{1}^{W}\right)\right|^{2}+2 \alpha_{2}\left(\left(\boldsymbol{C}_{m} \boldsymbol{x}_{2}^{W^{2}}\right)\left(\boldsymbol{C}_{m} \boldsymbol{x}_{1}^{W}\right)^{*}\right.\right. \\
& \left.\left.+\left(\boldsymbol{C}_{m} \boldsymbol{x}_{1}^{W}\right)\left(\boldsymbol{C}_{m} \boldsymbol{x}_{2}^{|W|^{2}}\right)\right)\right] e^{-\mathrm{i} \omega^{\mathrm{c}} \tau}, \\
& \boldsymbol{F}_{3, m}^{W^{3}}=\boldsymbol{B}_{m} n^{c}\left(\boldsymbol{C}_{m} \boldsymbol{x}_{1}^{W}\right)\left(\alpha_{3}\left(\boldsymbol{C}_{m} \boldsymbol{x}_{1}^{W}\right)^{2}+2 \alpha_{2}\left(\boldsymbol{C}_{m} \boldsymbol{x}_{2}^{W^{2}}\right)\right) e^{-3 \mathrm{i} \omega^{\mathrm{c}} \tau}, \\
& \boldsymbol{F}_{3, m}^{|W|^{2} Z}=n^{c} \boldsymbol{B}_{m}\left[2 \alpha_{2}\left(\boldsymbol{C}_{m} \boldsymbol{x}_{1}^{Z}\right)\left(\boldsymbol{C}_{m} \boldsymbol{x}_{2}^{|W|^{2}}\right)+2 \alpha_{2}\left(\boldsymbol{C}_{m} \boldsymbol{x}_{1}^{W}\right)\left(\boldsymbol{C}_{m} \boldsymbol{x}_{2}^{Z W^{*}}\right)\right. \\
& \left.+2 \alpha_{2}\left(\boldsymbol{C}_{m} \boldsymbol{x}_{2}^{Z W}\right)\left(\boldsymbol{C}_{m} \boldsymbol{x}_{1}^{W}\right)^{*}+6 \alpha_{3}\left|\left(\boldsymbol{C}_{m} \boldsymbol{x}_{1}^{W}\right)\right|^{2}\left(\boldsymbol{C}_{m} \boldsymbol{x}_{1}^{Z}\right)\right] e^{-\mathrm{i} \omega^{\mathrm{c}} \tau} \\
& \boldsymbol{F}_{3, m}^{W^{2} Z^{*}}=n^{c} \boldsymbol{B}_{m}\left[2 \alpha_{2}\left(\boldsymbol{C}_{m} \boldsymbol{x}_{2}^{W^{2}}\right)\left(\boldsymbol{C}_{m} \boldsymbol{x}_{1}^{Z}\right)^{*}+2 \alpha_{2}\left(\boldsymbol{C}_{m} \boldsymbol{x}_{1}^{W}\right)\left(\boldsymbol{C}_{m} \boldsymbol{x}_{2}^{Z W^{*}}\right)^{*}\right. \\
& \left.+3 \alpha_{3}\left(\boldsymbol{C}_{m} \boldsymbol{x}_{1}^{Z}\right)^{*}\left(\boldsymbol{C}_{m} \boldsymbol{x}_{1}^{W}\right)^{2}\right] e^{-\mathrm{i} \omega^{\mathrm{c}} \tau}, \\
& \boldsymbol{F}_{3, m}^{W^{2} Z}=\boldsymbol{B}_{m} n^{c}\left(3 \alpha_{3}\left(\boldsymbol{C}_{m} \boldsymbol{x}_{1}^{W}\right)^{2}\left(\boldsymbol{C}_{m} \boldsymbol{x}_{1}^{Z}\right)+2 \alpha_{2}\left(\boldsymbol{C}_{m} \boldsymbol{x}_{2}^{W^{2}}\right)\left(\boldsymbol{C}_{m} \boldsymbol{x}_{1}^{Z}\right)\right. \\
& \left.+2 \alpha_{2}\left(\boldsymbol{C}_{m} \boldsymbol{x}_{2}^{Z W}\right)\left(\boldsymbol{C}_{m} \boldsymbol{x}_{1}^{W}\right)\right) e^{-3 \mathrm{i} \omega^{\mathrm{c}} \tau} .
\end{aligned}
$$

\section{Appendix C. Solvability conditions}

$$
\text { C.1. } \mathcal{O}\left(\epsilon^{3}\right) \text { : }
$$

The following two solvability conditions ensure that resonant (secular) forcing terms are eliminated and a uniform validity of the weakly nonlinear, asymptotic expansion of the steady-state solution is guaranteed: 


$$
\begin{aligned}
\left\langle\boldsymbol{x}_{1}^{W+},\right. & -\frac{\partial W}{\partial t_{2}} \mathcal{P} \boldsymbol{x}_{1}^{W}+-\frac{\partial Z}{\partial t_{2}} \mathcal{P} \boldsymbol{x}_{1}^{Z}+W\left(\sum_{m=1}^{N} \boldsymbol{F}_{3, m}^{W}\right)+|W|^{2} W\left(\sum_{m=1}^{N} \boldsymbol{F}_{3, m}^{|W|^{2} W}\right) \\
& +Z\left(\sum_{m=1}^{N} \boldsymbol{F}_{3, m}^{Z}\right)+|Z|^{2} Z\left(\sum_{m=1}^{N} \boldsymbol{F}_{3, m}^{|Z|^{2} Z}\right)+|W|^{2} Z\left(\sum_{m=1}^{N} \boldsymbol{F}_{3, m}^{|W|^{2} Z}\right) \\
& +|Z|^{2} W\left(\sum_{m=1}^{N} \boldsymbol{F}_{3, m}^{|Z|^{2} W}\right)+W^{2} Z^{*}\left(\sum_{m=1}^{N} \boldsymbol{F}_{3, m}^{W^{2} Z}\right) \\
& \left.+Z^{2} W^{*}\left(\sum_{m=1}^{N} \boldsymbol{F}_{3, m}^{Z^{2} W}\right)\right\rangle=0 \\
\left\langle\boldsymbol{x}_{1}^{Z+},\right. & -\frac{\partial W}{\partial t_{2}} \mathcal{P} \boldsymbol{x}_{1}^{W}+-\frac{\partial Z}{\partial t_{2}} \mathcal{P} \boldsymbol{x}_{1}^{Z}+W^{N}\left(\sum_{m=1}^{N} \boldsymbol{F}_{3, m}^{W}\right)+|W|^{2} W\left(\sum_{m=1}^{N} \boldsymbol{F}_{3, m}^{|W|^{2} W}\right) \\
& +Z\left(\sum_{m=1}^{N} \boldsymbol{F}_{3, m}^{Z}\right)+|Z|^{2} Z\left(\sum_{m=1}^{N} \boldsymbol{F}_{3, m}^{|Z|^{2} Z}\right)+|W|^{2} Z\left(\sum_{m=1}^{N} \boldsymbol{F}_{3, m}^{|W|^{2} Z}\right) \\
& +|Z|^{2} W\left(\sum_{m=1}^{N} \boldsymbol{F}_{3, m}^{|Z|^{2} W}\right)+W^{2} Z^{*}\left(\sum_{m=1}^{N} \boldsymbol{F}_{3, m}^{W W^{2} Z}\right) \\
& \left.+Z^{2} W^{*}\left(\sum_{m=1}^{N} \boldsymbol{F}_{3, m}^{Z^{2} W}\right)\right\rangle=0
\end{aligned}
$$

where $\mathcal{P}=\boldsymbol{I}+\tau \alpha_{1} n^{c} \sum_{m=1}^{N} \boldsymbol{B}_{m}\left(\boldsymbol{C}_{m} e^{-\mathrm{i} \omega^{\mathrm{c}} \tau}\right)$.

\section{REFERENCES}

Ananthrrishnan, N, Deo, Shardul \& Culick, Fred EC 2005 Reduced-order modeling and dynamics of nonlinear acoustic waves in a combustion chamber. Combustion Science and Technology 177 (2), 221-248.

Balasubramanian, K. \& Sujith, R.I. 2008 Thermoacoustic instability in a Rijke tube: Nonnormality and nonlinearity. Phys. Fluids 20 (4), 044103.

Bauerheim, M., Parmentier, J.-F., Salas, P., Nicoud, F. \& Poinsot, T. 2014 An analytical model for azimuthal thermoacoustic modes in an annular chamber fed by an annular plenum. Comb. \& Flame 161 (5), 1374-1389.

Bourgouin, J.-F., Durox, D., Moeck, J.P., Schuller, T. \& Candel, S. 2015 Characterization and modeling of a spinning thermoacoustic instability in an annular combustor equipped with multiple matrix injectors. J. Eng. Gas Turb. Power 137 (2), 021503.

CANDEL, S. 1992 Combustion instabilities coupled by pressure waves and their active control. In Symposium (International) on Combustion, , vol. 24, pp. 1277-1296. Elsevier.

Candel, S., Durox, D. \& Schuller, T. 2004 Flame interactions as a source of noise and combustion instabilities. In 10th AIAA/CEAS Aeroacoustics Conf., , vol. 2928, pp. 14441454 .

Candel, S., Maistret, E., Darabiha, N., Poinsot, T., Veynante, D. \& Lacas, F. 1988 Experimental and numerical studies of turbulent ducted flames. In Marble Symposium, CalTech, pp. 209-236.

Clavin, P., Pelcé, P. \& He, L. 1990 One-dimensional vibratory instability of planar flames propagating in tubes. J. Fluid Mech. 216, 299-322.

CULICK, FEC 1988 Combustion instabilities in liquid-fuelled propulsion systems . 
Culick, F.E.C. \& Kuentzmann, P. 2006 Unsteady motions in combustion chambers for propulsion systems. Tech. Rep.. DTIC Document.

Dowling, A.P. 1995 The calculation of thermoacoustic oscillations. J. Sound Vibr. 180 (4), $557-581$.

Ducruix, S., Schuller, T., Durox, D. \& Candel, S. 2005 Combustion instability mechanisms in premixed combustors. Progr. in Astron. \& Aeron. 210, 179.

Engelborghs, K., Luzyanina, T. \& Samaey, G. 2000 DDE-BIFTOOL: a Matlab package for bifurcation analysis of delay differential equations. TW Report 305.

Fedkiw, R.P., Aslam, T., Merriman, B. \& Osher, S. 1999 A non-oscillatory Eulerian approach to interfaces in multimaterial flows (the Ghost Fluid Method). J. Comp. Phys. 152 (2), 457-492.

Gelbert, G., Moeck, J.P., Paschereit, C.O. \& King, R. 2012 Feedback control of unstable thermoacoustic modes in an annular Rijke tube. Control Eng. Pract. 20 (8), 770-782.

George, K.J. \& Sujith, R.I. 2011 On Chu's disturbance energy. J. Sound Vibr. 330 (22), $5280-5291$.

Ghirardo, G, JuniPer, MP \& Moeck, JP 2016 Weakly nonlinear analysis of thermoacoustic instabilities in annular combustors. Journal of Fluid Mechanics 805, 52-87.

Ghirardo, Giulio, Juniper, Matthew P \& Moeck, Jonas P 2015 Stability criteria for standing and spinning waves in annular combustors. In ASME Turbo Expo 2015: Turbine Technical Conference and Exposition, pp. V04BT04A005-V04BT04A005. American Society of Mechanical Engineers.

HARRJE, D.T. 1972 Liquid propellant rocket combustion instability. Tech. Rep. NASA-SP-194. NASA.

Jahnke, Craig C \& Culick, Fred EC 1994 Application of dynamical systems theory to nonlinear combustion instabilities. Journal of propulsion and power 10 (4), 508-517.

JARLEBRING, E. 2008 The spectrum of delay-differential equations: computational analysis. $\mathrm{PhD}$ thesis, $\mathrm{PhD}$ thesis, Inst. Comp. Math., TU Braunschweig, Germany.

JEgAdEESAn, ViveKANANDAN \& SuJith, RI 2013 Experimental investigation of noise induced triggering in thermoacoustic systems. Proceedings of the Combustion Institute 34 (2), 3175-3183.

JuniPeR, M.P. 2011 Triggering in the horizontal Rijke tube: non-normality, transient growth and bypass transition. J. Fluid Mech. 667, 272-308.

JUNIPER, MP 2012 Weakly nonlinear analysis of thermoacoustic oscillations. 19th ICSV pp. $1-5$.

Kabiraj, Lipika \& Sujith, RI 2012 Nonlinear self-excited thermoacoustic oscillations: intermittency and flame blowout. Journal of Fluid Mechanics 713, 376-397.

Kopitz, J., Huber, A., Sattelmayer, T. \& Polifke, W. 2005 Thermoacoustic stability analysis of an annular combustion chamber with acoustic low order modeling and validation against experiment. In ASME Turbo Expo 2005: Power for Land, Sea, and Air, pp. 583-593. Amer. Soc. Mech. Eng.

Laera, D, CAmpa, G \& CAmporeale, SM 2017 A finite element method for a weakly nonlinear dynamic analysis and bifurcation tracking of thermo-acoustic instability in longitudinal and annular combustors. Applied Energy 187, 216-227.

LEE, D.S. \& Anderson, T.J. 1999 Measurements of fuel/air-acoustic coupling in lean premixed combustion systems. In AIAA, Aerosp. Sci. Meeting and Exhibit, Reno, NV.

Lee, D.-H. \& Lieuwen, T.C. 2003 Premixed flame kinematics in a longitudinal acoustic field. J. Prop. Power 19 (5), 837-846.

Lieuwen, T.C. \& YANG, V. 2005 Combustion instabilities in gas turbine engines (operational experience, fundamental mechanisms and modeling). Prog. Astron. Aeron. .

Mensah, G.A. \& Moeck, J.P. 2015 Efficient computation of thermoacoustic modes in annular combustion chambers based on Bloch-wave theory. In ASME Turbo Expo 2015: Turbine Technical Conference and Exposition, p. 43476. Amer. Soc. Mech. Eng.

Moeck, J.P., Paul, M. \& Paschereit, C.O. 2010 Thermoacoustic instabilities in an annular Rijke tube. In ASME Turbo Expo 2010: Power for Land, Sea, and Air, pp. 1219-1232. Amer. Soc. Mech. Eng.

Newell, Alan C \& Whitehead, John A 1969 Finite bandwidth, finite amplitude convection. Journal of Fluid Mechanics 38 (2), 279-303. 
Nicoud, F. \& Poinsot, T. 2005 Thermoacoustic instabilities: Should the Rayleigh criterion be extended to include entropy changes? Comb. \& Flame 142 (1), 153-159.

Noiray, Nicolas, Durox, Daniel, Schuller, Thierry \& Candel, SÉbastien 2008 A unified framework for nonlinear combustion instability analysis based on the flame describing function. Journal of Fluid Mechanics 615, 139-167.

Oden, J Tinsley \& Demkowicz, Leszek 2010 Applied functional analysis. CRC press.

Oden, J Tinsley \& Demkowicz, Leszek 2017 Applied functional analysis. CRC press.

Orchini, Alessandro, Rigas, Georgios \& Juniper, Matthew P 2016 Weakly nonlinear analysis of thermoacoustic bifurcations in the rijke tube. Journal of Fluid Mechanics 805, $523-550$.

Parmentier, J.-F., Salas, P., Wolf, P., Staffelbach, G., Nicoud, F. \& Poinsot, T. 2012 A simple analytical model to study and control azimuthal instabilities in annular combustion chambers. Comb. \& Flame 159 (7), 2374-2387.

Poinsot, T., Trouve, A.C., Veynante, D.P., Candel, S. \& Esposito, E.J. 1987 Vortexdriven acoustically coupled combustion instabilities. J. Fluid Mech. 177, 265-292.

Poinsot, T. \& Veynante, D. 2005 Theoretical and Numerical Combustion. RT Edwards, Inc.

Polifke, W., Paschereit, C.O. \& Döbbeling, K. 2001 Constructive and destructive interference of acoustic and entropy waves in a premixed combustor with a choked exit. Int. J. Acoust. Vib 6 (3), 135-146.

Salas, Pablo 2013 Aspects numériques et physiques des instabilités thermoacoustiques dans les chambres de combustion annulaire. PhD thesis, Ph. D. thesis, Université Bordeaux I, Bordeaux, France.

Sattelmayer, T. \& Polifke, W. 2003 Assessment of methods for the computation of the linear stability of combustors. Comb. Sci. Techn. 175 (3), 453-476.

Sayadi, T., Le Chenadec, V., Schmid, P.J., Richecoeur, F. \& Massot, M. 2014 Thermoacoustic instability - a dynamical system and time domain analysis. J. Fluid Mech. 753, 448-471.

Schmid, P.J. \& Henningson, D.S. 2001 Stability and Transition in Shear Flows. Springer Verlag, New York.

Schmid, Peter J, de Pando, Miguel Fosas \& Peake, Nigel 2017 Stability analysis for n-periodic arrays of fluid systems. Physical Review Fluids 2 (11), 113902.

Sensiau, C., Nicoud, F. \& Poinsot, T. 2008 Computation of azimuthal combustion instabilities in an helicopter combustion chamber. AIAA paper 2947.

Sipp, Denis \& LeBedev, Anton 2007 Global stability of base and mean flows: a general approach and its applications to cylinder and open cavity flows. Journal of Fluid Mechanics 593, 333-358.

Staffelbach, G., Gicquel, L.Y.M., Boudier, G. \& Poinsot, T. 2009 Large eddy simulation of self excited azimuthal modes in annular combustors. Proc. Comb. Inst. 32 (2), 29092916.

Stow, S.R. \& Dowling, A.P. 2001 Thermoacoustic oscillations in an annular combustor. In ASME Turbo Expo 2001: Power for Land, Sea, and Air, p. 0037. Amer. Soc. Mech. Eng.

Subramanian, P., Sujith, R.I. \& Wahi, P. 2013 Subcritical bifurcation and bistability in thermoacoustic systems. J. Fluid Mech. 715, 210-238.

Sujith, RI, JuniPeR, MP \& Schmid, PJ 2016 Non-normality and nonlinearity in thermoacoustic instabilities. International Journal of Spray and Combustion Dynamics 8 (2), 119-146.

Wolf, P., Staffelbach, G., Gicquel, L.Y.M., Müller, J.-D. \& Poinsot, T. 2012 Acoustic and large eddy simulation studies of azimuthal modes in annular combustion chambers. Comb. \& Flame 159 (11), 3398-3413.

Worth, N.A. \& Dawson, J.R. 2013a Modal dynamics of self-excited azimuthal instabilities in an annular combustion chamber. Comb. \& Flame 160 (11), 2476-2489.

Worth, N.A. \& Dawson, J.R. $2013 b$ Self-excited circumferential instabilities in a model annular gas turbine combustor: Global flame dynamics. Proc. Comb. Inst. 34 (2), 31273134. 